Article

\title{
Chemical Constituent Profiling of Paecilomyces cicadae Liquid Fermentation for Astragli Radix
}

\author{
Yuqi Wang ${ }^{1}$, Xiaodan Mei ${ }^{1}$, Zihan Liu ${ }^{1}$, Jie Li ${ }^{1}$, Xiaoxin Zhang ${ }^{1}$, Shaoping Wang ${ }^{2}$, \\ Zikai Geng ${ }^{2}$, Long Dai ${ }^{2, *}$ and Jiayu Zhang ${ }^{1,2, *}$ \\ 1 School of Chinese Pharmacy, Beijing University of Chinese Medicine, Beijing 100029, China \\ 2 School of Pharmacy, BIN ZHOU Medical University, Yantai 260040, China \\ * Correspondence: druglab@sina.com (L.D.); zhangjiayu0615@163.com (J.Z.); Tel.: +86-10-0535-6913719 (L.D.); \\ +86-10-0535-6913719 (J.Z.)
}

Received: 1 August 2019; Accepted: 12 August 2019; Published: 14 August 2019

\begin{abstract}
Astragli Radix (AR) is one of the most popular traditional Chinese medicines with chemical constituents including flavonoids and saponins. As recently evidenced, some fungi or their fermentation liquid may have the potential to affect the bioactive constituents and different pharmacological effects of AR. Thus, the composition of fermented AR (FAR) produced by Paecilomyces cicadae (Miquel) Samson in liquid-state fermentation was investigated using a UHPLC-LTQ-Orbitrap mass spectrometer in both positive and negative ion modes. Firstly, the MS ${ }^{\mathrm{n}}$ data sets were obtained based on a data-dependent acquisition method and a full scan-parent ions list-dynamic exclusion (FS-PIL-DE) strategy. Then, diagnostic product ions (DPIs) and neutral loss fragments (NLFs) were proposed for better constituent detection and structural characterization. Consequently, 107 constituents in total, particularly microconstituents in FAR and AR, were characterized and compared in parallel on the same LTQ-Orbitrap instrument. Our results indicated that AR fermentation with Paecilomyces significantly influenced the production of saponins and flavonoids, especially increasing the content of astragaloside IV. In conclusion, this research was not only the first to show changes in the chemical components of unfermented AR and FAR, but it also provides a foundation for further studies on the chemical interaction between microbiota and AR.
\end{abstract}

Keywords: Astragli Radix; Paecilomyces cicadae; liquid fermentation; chemical constituents; UHPLC-LTQ-Orbitrap MS

\section{Introduction}

Over the past several decades, accompanied by growing demand for traditional Chinese medicines (TCMs) and a gradual reduction of wild resources, improving the content of active ingredients and cultivating new varieties with high quality have become the most urgent tasks in the development of herbal resources. Recently, the application of TCMs by submerged fermentation of edible and pharmaceutical fungi has become a hot issue which opens up broad prospects for TCMs. Previous studies have shown that medicinal fungi can secrete important secondary metabolic products which degrade macromolecular material into small molecules [1,2]. By means of fermentation, TCMs can improve intrinsic conversion efficiency and new compound growth rates for increased therapeutic effect. Besides this, fermentation can also reduce the toxicity of TCMs containing typical compounds such as alkaloids, lactones, toxic glycosides, toxic proteins, anthraquinones, tannins, and heavy metals [3,4].

Paecilomyces cicadae (Miquel) Samson, as an entomogenous and medicinal fungus, is thought to be the anamorph stage of Cordyceps cicadae Shing. It is widely used as a tonic for nourishment as well as a 
functional food, and it has attracted considerable attention due to its wide range of nutritional and pharmacological activities, including immunomodulatory [5], antioxidation, anti-aging, anti-tumor [6], and anti-inflammation activity and ameliorating renal function [7].

Astragli Radix (AR), known as Huangqi in Chinese, is one of the most widely used traditional herbal medicines. It is the dried root of Astragalus membranaceus (Fisch.) Bge. var. mongholicus (Bge.) Hsiao or Astragalus membranaceus (Fisch.) Bge. AR contains relatively high quantities of saponins, flavonoids, polysaccharides, and some trace elements, which are known for their antibacterial, anti-inflammatory, analgesic, anti-cancer, anti-oxidant, and other pharmacological effects [8-11]. However, different processing methods (such as fermentation) may change the properties of this material.

Many studies have reported that natural macromolecular compounds exist in herbal medicines, including polysaccharides, flavonoid glycosides, and saponins, which promote pharmacological anti-tumor, anti-oxidant, and anti-inflammatory effects. However, most herbal macromolecular compounds cannot be digested and used by the human being in the absence of microbial fermentation [12]. For example, polysaccharides fermented by microbiota can be converted into short-chain fatty acids, which are easily digested and absorbed by the human body [13]. In addition, when red ginseng is fermented by Bifidobacterium $\mathrm{H}-1, \mathrm{Rg} 3$ is transformed to Rh2, which has exhibited potent cytotoxicity against tumor cells [14]. Therefore, the aim of our present study was to develop liquid-state fermentation for AR by Paecilomyces cicadae (Miquel) Samson and to investigate whether this method leads to changes in the components contained in AR.

In order to obtain comprehensive knowledge of the compounds in the fermented AR (FAR), we further characterized its chemical constituents by way of ultra-high-performance liquid chromatography coupled with high-resolution mass spectrometry (UHPLC-HRMS). Moreover, the application of a full scan-parent ions list-dynamic exclusion (FS-PIL-DE) strategy coupled with diagnostic product ions (DPIs) and neutral loss fragments (NLFs) is proposed for better constituent detection and structural characterization $[15,16]$. Finally, the constituents, particularly microconstituents in FAR and AR, were characterized and compared in parallel on the same LTQ-Orbitrap instrument.

\section{Results}

\subsection{Establishment of the Analytical Strategy}

In this study, a comprehensive and effective strategy is proposed to systematically screen and identify compounds on a UHPLC-LTQ-Orbitrap MS instrument. The analytic strategy roughly consisted of three steps. The first step was online data acquisition. A full mass scan was performed with a resolution of 30,000. Meanwhile, high-resolution extracted ion chromatography (HREIC) was adopted to extract the candidates from the high-quality, accurate raw mass data both in negative and positive ion modes. Secondly, PIL-DE and data-dependent acquisition methods were employed to obtain specific ESI-MS/MS datasets based on those screened candidates. Then, DPI and NLF techniques were used as supplementary tools for the selective detection of constituents that possess similar mass fragmentation behaviors to those of reference standards. Finally, the structures of the compounds were elucidated according to the accurate mass measurement, fragmentation patterns, diagnostic product ions, and literature reports. The general procedures of our strategy and approach are summarized in the diagram shown in Figure 1. 


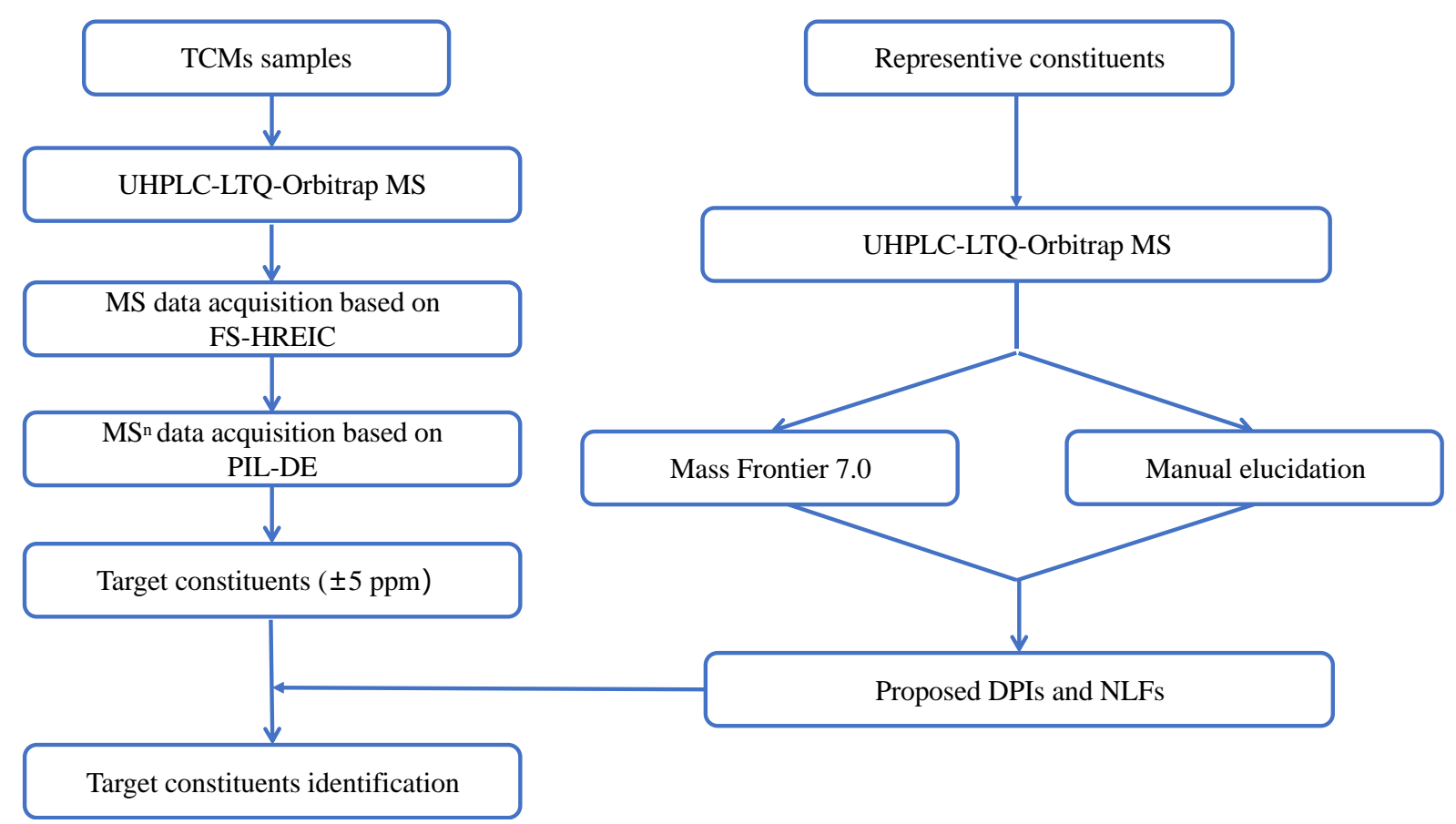

Figure 1. Summary diagram of the presently developed strategy and methodology.

\subsection{Establishment of the Data Acquisition Methods}

By employing the full scan method, abundant data were generated with large amounts of invalid data. Thus, to reduce potential disturbances by irrelevant substances and avoid missing target drug compounds (especially trace compounds), HREIC was developed for compound detection [17]. The application of HREIC could intelligently filter the background and matrix-related ions from drug-related ions according to the accurate mass of $[\mathrm{M}-\mathrm{H}]^{-}$or $[\mathrm{M}+\mathrm{H}]^{+}$ions. The molecular weights and elemental compositions of compounds derived from the accurate mass measurements can also be readily predicted. As a result, the lower level of target compounds can be captured clearly. For a complicated system, FS was not an appropriate approach to obtain the entire MS/MS dataset due to the numerous potential candidates. Therefore, the PIL-DE method served as a supplementary method to obtain the MS/MS fragmentation of the microconstituents [15]. By means of the PIL-DE method, MS/MS acquisition of predictable constituents that have the same molecular weights could be triggered due to its superior sensitivity and selectivity.

\subsection{Fragmentation Pattern Analysis and DPI Determination}

To facilitate the structural elucidation of constituents in AR and FAR, sixteen standards, including eight astragalus saponins and eight flavonoids, were subsequently analyzed by UHPLC-LTQ-Orbitrap MS. All the standards exhibited $[\mathrm{M}-\mathrm{H}]^{-}$or $[\mathrm{M}+\mathrm{H}]^{+}$ions of sufficient intensity that could be isolated automatically and subjected to collision induced dissociation (CID)-MS/MS analysis. Mass Frontier v7.0 software (Thermo Scientific, Waltham, MA, USA) and manual elucidation were used to acquire comprehensive structural identification of these reference compounds.

In CID mode, compounds are often divided into two parts, such as product ions (emerge in ESI-MS/MS spectra due to their property of being easily ionized) and neutral fragments (observed in ESI-MS/MS spectra due to their mass difference and neutral characteristics) $[18,19]$, which are complementary in structure. It is well documented that compounds with similar backbone structure exhibit comparable fragmentation patterns, resulting in certain diagnostic product ions (DPIs) and regular neutral loss fragments (NLFs). Consequently, the combination of DPIs and NLFs was helpful to rapidly performing the structural elucidation [20,21]. 
Eight astragalus saponin standards were subsequently analyzed firstly in the CID-MS/MS experiment. For instance, astragaloside I, isoastragaloside I, astragaloside II, isoastragaloside II, and astragaloside IV possess the same backbone structure, and their differences are limited to the quantity and position of acetyl groups connected to xylose. For instance, there are two acetyl groups on the 2 and 3 positions of xylose in astragaloside I, one acetyl group on the 2 position of xylose in astragaloside II, and zero acetyl groups in astragaloside IV. By comparing the MS/MS spectra of their product ions, some characteristic dissociation pathways of astragalus saponins could be summarized, which provided a basis for further characterization of the other candidates. Taking the negative ion mode as an example, all of the deprotonated ions could lose one glucosyl $\left(\mathrm{C}_{6} \mathrm{H}_{10} \mathrm{O}_{5} \cdot\right)$ or xylose $\left(\mathrm{C}_{5} \mathrm{H}_{8} \mathrm{O}_{4} \cdot\right)$ or even both of them in their ESI-MS spectra. Then, the base peak ions of [M-H-162 $]^{-},[\mathrm{M}-\mathrm{H}-132]^{-}$, and [M-H-294] ${ }^{-}$ could be formed. Owing to the special structure of the acetyl group (Ac), other characteristic fragment ions were also generated by the loss of $42(\mathrm{Ac}), 60\left(\mathrm{Ac}+\mathrm{H}_{2} \mathrm{O}\right)$, and $84(2 \mathrm{Ac})$. These diagnostic product ions could be employed to ascertain the structural skeletons of astragalus saponins and simplify the following structural elucidation.

In addition, we also selected eight flavonoids as subjects to determine their DPIs. Owing to the special structures of flavonoid glycosides, the base peak ion of [M-H-162] ${ }^{-}$was usually produced via the loss of the glucose moiety in their ESI-MS² spectra. Meanwhile, the other characteristic ions at [M-H-15] $]^{-},[\mathrm{M}-\mathrm{H}-18]^{-},[\mathrm{M}-\mathrm{H}-28]^{-},[\mathrm{M}-\mathrm{H}-29]^{-},[\mathrm{M}-\mathrm{H}-31]^{-},[\mathrm{M}-\mathrm{H}-43]^{-},[\mathrm{M}-\mathrm{H}-44]^{-}$, and $[\mathrm{M}-\mathrm{H}-61]^{-}$were yielded by losing $\mathrm{CH}_{3}, \mathrm{H}_{2} \mathrm{O}, \mathrm{CO}, \mathrm{HCO}, \mathrm{OCH}_{3}, \mathrm{CH}_{3}+\mathrm{CO}, \mathrm{HCO}+\mathrm{CH}_{3}$, and $\mathrm{H}_{2} \mathrm{O}+\mathrm{CO}+\mathrm{CH}_{3}$ in negative mode. Therefore, the DPIs mentioned above could be utilized for deducing the structures of related compounds from abundant complex constituents.

\subsection{Structural Assignment of Chemical Constituents in AR and FAR}

Saponins and flavonoids are the major chemical constituents in AR. As a result, 107 compounds in total were detected and characterized from AR and FAR by way of UHPLC-LTQ-Orbitrap MS with the established strategy. Among these compounds, 42 were attributed to saponins while the remaining 65 were identified as flavonoids. The correlative data are summarized in Tables 1 and 2, and the HREIC spectra of detected constituents are illustrated in Figure 2. The fragmentation patterns of representative saponins and flavonoids are shown in Figures S1 and S2. 
Table 1. Summary of identified saponins in Astragli Radix (AR) and fermented AR (FAR).

\begin{tabular}{|c|c|c|c|c|c|c|c|c|c|c|}
\hline Peak & $\begin{array}{c}t_{R} \\
/ \min \end{array}$ & Ion Mode & Formula & $\begin{array}{l}\text { Theoretical } \\
\text { Mass } m / z\end{array}$ & $\begin{array}{l}\text { Experimental } \\
\text { Mass } m / z\end{array}$ & Error (ppm) & $\mathrm{MS}^{2} / \mathrm{MS}^{3}$ Fragment Ions & Identification & FAR & AR \\
\hline A1 & 7.57 & $\mathrm{P}$ & $\mathrm{C}_{48} \mathrm{H}_{79} \mathrm{O}_{18}$ & 943.52664 & 943.52582 & -0.288 & $\mathrm{MS}^{2}[943]: 925(100), 927(76), 486(30), 859(13), 927(13), 845(10), 827(6)$ & Soyasaponin I/isomer & - & + \\
\hline A2 & 9.32 & $\mathrm{P}$ & $\mathrm{C}_{43} \mathrm{H}_{71} \mathrm{O}_{15}$ & 827.47875 & 827.47443 & -3.218 & $\mathrm{MS}^{2}[827]: 709(100), 809(10), 691(9), 768(4), 737(2), 695(2), 577(2), 335(2), 467(1)$ & $\begin{array}{c}\text { Acetylastragaloside II } \\
\text { isomer }\end{array}$ & + & ++ \\
\hline A3 & 10.43 & $\mathrm{P}$ & $\mathrm{C}_{38} \mathrm{H}_{63} \mathrm{O}_{11}$ & 695.43704 & 695.43274 & -3.391 & $\mathrm{MS}^{2}[695]: 577(100), 677(12), 559(9), 583(5), 576(2)$ & Mongholicoside II & + & ++ \\
\hline \multirow{2}{*}{ A4 } & \multirow{2}{*}{10.76} & $\mathrm{~N}$ & $\mathrm{C}_{47} \mathrm{H}_{77} \mathrm{O}_{19}$ & 945.50700 & 945.50916 & 4.023 & $\begin{array}{l}\mathrm{MS}^{2}[945]: 783(100), 489(3), 621(2), 765(1), 651(1) \\
\text { MS }^{3}[783]: 489(100), 621(53), 383(35), 651(26)\end{array}$ & Agroastragaloside $\mathrm{V}$ & + & ++ \\
\hline & & $\mathrm{P}$ & $\mathrm{C}_{47} \mathrm{H}_{79} \mathrm{O}_{19}$ & 947.52155 & 947.52026 & -0.788 & 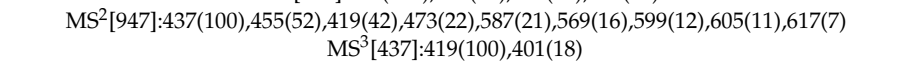 & & & \\
\hline A5 & 11.61 & $\mathrm{~N}$ & $\mathrm{C}_{41} \mathrm{H}_{69} \mathrm{O}_{14}$ & 785.46983 & 785.47198 & 4.834 & $\operatorname{MS}^{2}[785]: 491(100), 623(26), 489(15), 435(6), 649(6), 717(4), 741(1)$ & $\begin{array}{l}\text { Cyclocanthoside } \\
\text { E/isomer }\end{array}$ & + & ++ \\
\hline $\mathrm{A} 6^{*}$ & 11.79 & $\mathrm{~N}$ & $\mathrm{C}_{41} \mathrm{H}_{67} \mathrm{O}_{14}$ & 783.45363 & 783.45612 & 4.578 & $\operatorname{MS}^{2}[783]: 489(100), 621(46), 651(36), 383(15), 453(11), 515(8), 471(6)$ & Isoastragaloside IV & ++ & + \\
\hline A7 & 11.82 & $\mathrm{~N}$ & $\mathrm{C}_{42} \mathrm{H}_{69} \mathrm{O}_{15}$ & 813.46474 & 813.46747 & 3.375 & $\mathrm{MS}^{2}[813]: 745(100), 767(67), 771(63), 607(37), 651(20), 387(25)$ & $\begin{array}{l}\text { Astramembranoside } \\
\text { A/isomer }\end{array}$ & + & ++ \\
\hline A8 & 11.89 & $\mathrm{~N}$ & $\mathrm{C}_{41} \mathrm{H}_{69} \mathrm{O}_{14}$ & 785.46983 & 785.46277 & -4.892 & $\operatorname{MS}^{2}[785]: 491(100), 623(58), 489(44), 653(30), 717(21), 737(20)$ & $\begin{array}{l}\text { Cyclocanthoside } \\
\text { E/isomer }\end{array}$ & + & ++ \\
\hline A9 & 12.42 & $\begin{array}{l}\mathrm{N} \\
\mathrm{P}\end{array}$ & $\begin{array}{l}\mathrm{C}_{42} \mathrm{H}_{69} \mathrm{O}_{15} \\
\mathrm{C}_{42} \mathrm{H}_{71} \mathrm{O}_{15}\end{array}$ & $\begin{array}{l}813.46474 \\
815.47930\end{array}$ & $\begin{array}{l}813.46729 \\
815.47729\end{array}$ & $\begin{array}{l}3.154 \\
-1.788\end{array}$ & $\begin{array}{c}\mathrm{MS}^{2}[813]: 651(100), 687(64), 745(47), 767(44), 473(26), 707(23) \\
\left.\left.\text { MS }^{2}[815]: 437(100), 455(48), 419(41), 473(29), 599(20), 653(7), 278\right), 463\right), 437(6), 467(6), 745(5) \\
\text { MS }^{3}[437]: 419(100), 351(26), 175(22), 215(16), 253(16), 167(10)\end{array}$ & $\begin{array}{l}\text { Astramembranoside } \\
\text { A/isomer }\end{array}$ & + & ++ \\
\hline A10 & 12.55 & $\mathrm{~N}$ & $\mathrm{C}_{41} \mathrm{H}_{69} \mathrm{O}_{14}$ & 785.46983 & 785.47180 & 4.605 & $\mathrm{MS}^{2}[785]: 491(100), 623(24), 717(13), 747(4), 629(4), 701(3)$ & $\begin{array}{l}\text { Cyclocanthoside } \\
\text { E/isomer }\end{array}$ & - & ++ \\
\hline A11 & 12.59 & $\mathrm{~N}$ & $\mathrm{C}_{43} \mathrm{H}_{69} \mathrm{O}_{15}$ & 825.46419 & 825.46735 & 3.151 & $\mathrm{MS}^{2}[825]: 765(100), 783(45), 757(17), 787(12), 779(11), 673(5), 401(4)$ & $\begin{array}{l}\text { Astragaloside II } \\
\text { isomer }\end{array}$ & + & ++ \\
\hline A12 & 13.32 & $\mathrm{~N}$ & $\mathrm{C}_{36} \mathrm{H}_{61} \mathrm{O}_{11}$ & 669.42248 & 669.42383 & 4.468 & $\mathrm{MS}^{2}[669]: 623(100), 533(46), 465(39), 367(29), 651(18)$ & $\begin{array}{l}\text { Mongholicoside A } \\
\text { /isomer }\end{array}$ & + & ++ \\
\hline A13 & 13.79 & $\mathrm{~N}$ & $\mathrm{C}_{43} \mathrm{H}_{71} \mathrm{O}_{15}$ & 827.48039 & 827.48138 & 3.181 & $\mathrm{MS}^{2}[827]: 759(100), 767(39), 784(36), 357(34), 781(33), 616(24) 785(22), 770(20)$ & Agroastragaloside II & + & ++ \\
\hline A14 & 14.00 & $\mathrm{~N}$ & $\mathrm{C}_{43} \mathrm{H}_{69} \mathrm{O}_{15}$ & 825.46419 & 825.46710 & 4.849 & $\mathrm{MS}^{2}[825]: 633(100), 763(38), 765(34), 645(27), 486(17), 643(15), 783(15)$ & $\begin{array}{l}\text { Astragaloside II } \\
\text { isomer }\end{array}$ & - & + \\
\hline A15 & 14.07 & $\mathrm{~N}$ & $\mathrm{C}_{36} \mathrm{H}_{61} \mathrm{O}_{11}$ & 669.42248 & 669.42377 & 4.378 & $\mathrm{MS}^{2}[669]: 623(100), 601(57), 397(26), 533(21), 601(20), 625(19), 641(17), 651(15)$ & $\begin{array}{l}\text { Mongholicoside A } \\
\text { /isomer }\end{array}$ & + & ++ \\
\hline A16* & 14.27 & $\mathrm{~N}$ & $\mathrm{C}_{41} \mathrm{H}_{67} \mathrm{O}_{14}$ & 783.45363 & 783.45813 & 4.144 & $\operatorname{MS}^{2}[783]: 489(100), 621(14), 383(11), 453(4)$ & Astragaloside IV & ++ & + \\
\hline A17 & 14.55 & $\mathrm{~N}$ & $\mathrm{C}_{41} \mathrm{H}_{69} \mathrm{O}_{14}$ & 785.46983 & 785.46313 & -4.433 & MS $^{2}[785]: 490(100), 489(79), 491(28), 623(16), 383(13), 621(11)$ & $\begin{array}{l}\text { Cyclocanthoside } \\
\text { E/isomer }\end{array}$ & + & ++ \\
\hline A18* & 14.59 & $\mathrm{~N}$ & $\mathrm{C}_{41} \mathrm{H}_{67} \mathrm{O}_{14}$ & 783.45363 & 783.45654 & 3.115 & $\mathrm{MS}^{2}[783]: 489(100), 383(13), 621(12), 453(4), 401(2), 472(2), 381(2)$ & Astragaloside III & ++ & + \\
\hline A19 & 16.10 & $\begin{array}{l}\mathrm{N} \\
\mathrm{P}\end{array}$ & $\begin{array}{l}\mathrm{C}_{51} \mathrm{H}_{81} \mathrm{O}_{21} \\
\mathrm{C}_{51} \mathrm{H}_{83} \mathrm{O}_{21}\end{array}$ & $\begin{array}{l}1029.52758 \\
1031.54214\end{array}$ & $\begin{array}{l}1029.52173 \\
1031.54199\end{array}$ & $\begin{array}{l}-4.619 \\
-0.141\end{array}$ & $\begin{array}{c}\operatorname{MS}^{2}[1029]: 985(100), 984(18), 967(2) \\
\operatorname{MS}^{2}[1031]: 984(100), 494(57), 558(52), 331(50), 667(49), 936(48), 323(47), 482(46), 300(45)\end{array}$ & Agroastragaloside III & + & ++ \\
\hline A20 & 16.29 & $\mathrm{~N}$ & $\mathrm{C}_{48} \mathrm{H}_{77} \mathrm{O}_{18}$ & 941.51209 & 941.50549 & -5.259 & $\mathrm{MS}^{2}[941]: 923(100), 524(56), 873(36), 923(32), 615(27), 523(26), 879(20), 456(18)$, & \multirow{2}{*}{$\begin{array}{c}\text { Soyasaponin I/isomer } \\
\text { Acetylastragaloside I } \\
\text { /isomer }\end{array}$} & + & ++ \\
\hline A21 & 16.31 & $\mathrm{~N}$ & $\mathrm{C}_{47} \mathrm{H}_{73} \mathrm{O}_{17}$ & 909.48532 & 909.48804 & 4.192 & $\operatorname{MS}^{2}[909]: 891(100), 523(50), 569(49), 613(49), 455(35), 701(31), 435(18), 757(16)$ & & + & ++ \\
\hline A22* & 16.67 & $\begin{array}{l}\mathrm{N} \\
\mathrm{P}\end{array}$ & $\begin{array}{l}\mathrm{C}_{43} \mathrm{H}_{69} \mathrm{O}_{15} \\
\mathrm{C}_{43} \mathrm{H}_{71} \mathrm{O}_{15}\end{array}$ & $\begin{array}{l}825.46419 \\
827.47875\end{array}$ & $\begin{array}{l}825.46796 \\
827.47729\end{array}$ & $\begin{array}{c}3.890 \\
-1.762\end{array}$ & $\begin{array}{c}\text { MS'}^{2}[825]: 765(100), 633(17), 621(11), 461(10), 489(8) \\
\text { MS'}^{2}[827]: 269(100), 592(67), 629(66), 351(64), 296(63), 633(60), 709(60), 247(59), 277(57)\end{array}$ & Astragaloside II & ++ & + \\
\hline A23 & 16.74 & $\mathrm{~N}$ & $\mathrm{C}_{36} \mathrm{H}_{59} \mathrm{O}_{11}$ & 667.40683 & 667.40820 & 4.512 & MS $^{2}[667]: 649(100), 449(82), 621(81), 299(80), 450(74), 485(54)$ & Mongholicoside B & + & ++ \\
\hline \multirow[t]{2}{*}{ A24 } & \multirow{2}{*}{16.85} & $\mathrm{~N}$ & $\mathrm{C}_{48} \mathrm{H}_{77} \mathrm{O}_{18}$ & 941.51209 & 941.51392 & 3.694 & $\begin{array}{l}\text { MS }^{2}[941]: 923(100), 525(73), 615(51), 744(49), 879(41), 457(40), 795(37), 437(35), 597(16) \\
\text { MS }^{3}[923]: 525(100), 733(55), 879(47), 437(44), 597(28), 457(21)\end{array}$ & \multirow[t]{2}{*}{ Soyasaponin I/isomer } & \multirow[t]{2}{*}{+} & \multirow[t]{2}{*}{++} \\
\hline & & $\mathrm{P}$ & $\mathrm{C}_{48} \mathrm{H}_{79} \mathrm{O}_{18}$ & 943.52664 & 943.52496 & -1.200 & $\operatorname{MS}^{2}[943]: 599(100), 797(88), 441(79), 423(48), 617(28), 581(23), 520(10), 269(8), 454(8), 867(8), 448(8)$ & & & \\
\hline A25 & 16.91 & $\mathrm{~N}$ & $\mathrm{C}_{43} \mathrm{H}_{69} \mathrm{O}_{15}$ & 825.46419 & 825.46631 & 3.892 & MS $^{2}[825]: 783(100), 607(32), 765(30), 735(16), 795(10), 697(9), 758(7)$ & $\begin{array}{l}\text { Astragaloside II } \\
\text { isomer }\end{array}$ & - & + \\
\hline A26 & 17.53 & $\mathrm{~N}$ & $\mathrm{C}_{42} \mathrm{H}_{69} \mathrm{O}_{15}$ & 813.46474 & 813.46686 & 4.625 & $\mathrm{MS}^{2}[813]: 767(100), 274(73), 677(18)$ & $\begin{array}{l}\text { Astramembranoside } \\
\text { A/isomer }\end{array}$ & + & ++ \\
\hline
\end{tabular}


Table 1. Cont

\begin{tabular}{|c|c|c|c|c|c|c|c|c|c|c|}
\hline Peak & $\begin{array}{c}t_{R} \\
/ \min \end{array}$ & Ion Mode & Formula & $\begin{array}{l}\text { Theoretical } \\
\text { Mass } m / z\end{array}$ & $\begin{array}{l}\text { Experimental } \\
\text { Mass } m / z\end{array}$ & Error (ppm) & $\mathrm{MS}^{2} / \mathrm{MS}^{3}$ Fragment Ions & Identification & FAR & AR \\
\hline A27* & 18.12 & $\mathrm{~N}$ & $\mathrm{C}_{43} \mathrm{H}_{69} \mathrm{O}_{15}$ & 825.46419 & 825.46643 & 4.037 & $\mathrm{MS}^{2}[825]: 765(100), 633(19), 717(24), 495(20), 351(5)$ & Isoastragaloside II & - & + \\
\hline A28 & 18.80 & $\mathrm{~N}$ & $\mathrm{C}_{42} \mathrm{H}_{65} \mathrm{O}_{14}$ & 793.43853 & 793.44080 & 4.937 & $\operatorname{MS}^{2}[793]: 631(100), 725(24), 657(8), 724(7), 747(5), 718(5), 697(4)$ & $\begin{array}{l}\text { Huangqiyenin } \\
\text { E/isomer }\end{array}$ & - & + \\
\hline A29 & 18.83 & $\mathrm{~N}$ & $\mathrm{C}_{42} \mathrm{H}_{69} \mathrm{O}_{15}$ & 813.46474 & 813.46692 & 4.699 & $\operatorname{MS}^{2}[813]: 767(100), 677(11), 795(1)$ & $\begin{array}{l}\text { Astramembranoside } \\
\text { A/isomer }\end{array}$ & + & ++ \\
\hline A30 & 18.94 & N & $\mathrm{C}_{45} \mathrm{H}_{71} \mathrm{O}_{16}$ & 867.47476 & 867.47766 & 4.608 & $\mathrm{MS}^{2}[867]: 807(100), 765(61), 821(24), 731(24), 821(23)$ & $\begin{array}{l}\text { Astragaloside I } \\
\text { isomer }\end{array}$ & + & ++ \\
\hline A31 & 19.16 & $\mathrm{~N}$ & $\mathrm{C}_{47} \mathrm{H}_{73} \mathrm{O}_{17}$ & 909.48532 & 909.48846 & 4.654 & $\mathrm{MS}^{2}[909]: 891(100), 455(86), 569(33), 523(29), 613(28), 407(28), 763(26), 773(22)$ & $\begin{array}{l}\text { Acetylastragaloside } \\
\text { I/isomer }\end{array}$ & + & ++ \\
\hline A32 & 19.21 & $\mathrm{~N}$ & $\mathrm{C}_{48} \mathrm{H}_{77} \mathrm{O}_{18}$ & 941.51209 & 941.50427 & -4.555 & $\mathrm{MS}^{2}[941]: 923(100), 523(44), 879(37), 614(36), 523(36), 613(32), 732(31)$ & Soyasaponin I & + & ++ \\
\hline A33 & 19.34 & $\mathrm{~N}$ & $\mathrm{C}_{45} \mathrm{H}_{71} \mathrm{O}_{16}$ & 867.47476 & 867.47766 & 4.608 & $\mathrm{MS}^{2}[867]: 821(100), 799(34), 731(23), 343(16), 787(11)$ & $\begin{array}{l}\text { Astragaloside I } \\
\text { isomer }\end{array}$ & - & + \\
\hline A34 & 19.44 & $\mathrm{P}$ & $\mathrm{C}_{48} \mathrm{H}_{79} \mathrm{O}_{18}$ & 943.52664 & 943.52161 & -4.750 & $\mathrm{MS}^{2}[943]: 796(100), 598(88), 439(30), 597(27), 795(13)$ & Soyasaponin I/isomer & - & + \\
\hline A35 & 20.25 & $\mathrm{~N}$ & $\mathrm{C}_{42} \mathrm{H}_{65} \mathrm{O}_{14}$ & 793.43853 & 793.44073 & 4.849 & $\operatorname{MS}^{2}[793]: 455(100), 613(86), 435(85), 391(45)$ & $\begin{array}{l}\text { Huangqiyenin } \\
\text { E/isomer }\end{array}$ & - & + \\
\hline A36 & 20.34 & $\mathrm{~N}$ & $\mathrm{C}_{45} \mathrm{H}_{71} \mathrm{O}_{16}$ & 867.47476 & 867.47662 & 3.410 & $\mathrm{MS}^{2}[867]: 807(100), 799(52), 765(51), 731(44), 825(43), 731(29)$ & $\begin{array}{c}\text { Astragaloside I } \\
\text { isomer }\end{array}$ & - & + \\
\hline A37 $7^{*}$ & 20.95 & $\mathrm{~N}$ & $\mathrm{C}_{45} \mathrm{H}_{71} \mathrm{O}_{16}$ & 867.47476 & 867.47943 & 4.649 & $\mathrm{MS}^{2}[867]: 781(100), 807(55), 821(51), 765(18), 747(16)$ & Astragaloside I & ++ & + \\
\hline A38 & 22.12 & $\mathrm{~N}$ & $\mathrm{C}_{45} \mathrm{H}_{73} \mathrm{O}_{16}$ & 869.49096 & 869.49335 & 4.644 & $\mathrm{MS}^{2}[869]: 823(100), 801(46), 599(18), 785(15), 536(11), 731(10), 741(8), 705(8)$ & Agroastragaloside I & - & + \\
\hline A39* & 22.72 & $\mathrm{~N}$ & $\mathrm{C}_{45} \mathrm{H}_{71} \mathrm{O}_{16}$ & 867.47476 & 867.47711 & 3.974 & $\operatorname{MS}^{2}[867]: 807(100), 747(38), 685(29), 717(19), 765(16), 749(10)$ & Isoastragaloside I & - & + \\
\hline A40 & 22.77 & $\mathrm{~N}$ & $\mathrm{C}_{48} \mathrm{H}_{73} \mathrm{O}_{19}$ & 953.47570 & 953.47968 & 3.898 & $\begin{array}{c}\operatorname{MS}^{2}[953]: 909(100), 849(3), 867(2), 807(1) \\
\text { MS }^{3}[909]: 849(100), 807(87), 765(80), 867(56), 747(42)\end{array}$ & $\begin{array}{l}\text { Malonylastragaloside } \\
\text { I }\end{array}$ & + & ++ \\
\hline A42* & 23.79 & $\mathrm{~N}$ & $\mathrm{C}_{45} \mathrm{H}_{71} \mathrm{O}_{16}$ & 867.47476 & 867.47754 & 4.470 & $\mathrm{MS}^{2}[909]: 849(100), 867(27), 765(24), 801(14), 807(12), 747(11), 867(10)$ & $\begin{array}{c}\text { Acetylastragaloside } \\
\text { I/isomer } \\
\beta \text {-D-glucopyranoside, } \\
(3 \beta, 6 \alpha, 16 \beta, 20 \mathrm{R}, 24 \mathrm{~s})-3- \\
\text { [(3,4-di-O-acetyl- } \beta \text {-D- } \\
\text { xylopyranosyl)oxy]- } \\
\text { 20,24-epoxy- } \\
\text { 16,25-dihydroxy-9,19- } \\
\text { cyclolanostan-6-yl }\end{array}$ & + & ++ \\
\hline
\end{tabular}


Table 2. Summary of identified flavonoids in AR and FAR.

\begin{tabular}{|c|c|c|c|c|c|c|c|c|c|c|}
\hline Peak & $\begin{array}{c}\mathrm{t}_{\mathrm{R}} \\
/ \mathrm{min}\end{array}$ & Ion mode & Formula & $\begin{array}{l}\text { Theoretical } \\
\text { Mass } m / z\end{array}$ & $\begin{array}{l}\text { Experimental } \\
\text { Mass } m / z\end{array}$ & Error (ppm) & $\mathrm{MS}^{2} / \mathrm{MS}^{3}$ fragment ions & Identification & FAR & AR \\
\hline B1 & 4.37 & $\mathrm{~N}$ & $\mathrm{C}_{29} \mathrm{H}_{37} \mathrm{O}_{16}$ & 641.20926 & 641.21063 & 4.708 & $\mathrm{MS}^{2}[641]: 479(100), 317(75), 595(35), 611(30), 623(26), 379(24), 610(22)$ & $\begin{array}{l}5^{\prime} \text {-hydroxy-isomucronulatol- } \\
2^{\prime}, 5^{\prime} \text {-di-o-glucoside }\end{array}$ & + & ${ }^{++}$ \\
\hline B2 & 4.47 & $\mathrm{P}$ & $\mathrm{C}_{24} \mathrm{H}_{25} \mathrm{O}_{12}$ & 505.13460 & 505.13318 & -1.727 & $\operatorname{MS}^{2}[505]: 333(100), 335(41), 373(26), 438(21), 281(21), 343(13), 282(11), 317(9), 181(7), 487(6)$ & Neocomplanoside/isomer & + & ++ \\
\hline B3 & 4.76 & $\mathrm{~N}$ & $\mathrm{C}_{28} \mathrm{H}_{31} \mathrm{O}_{16}$ & 623.16231 & 623.16388 & 3.165 & $\mathrm{MS}^{2}[623]: 299(100), 284(31), 604(7), 283(6), 456(6), 605(5), 443(5), 255(4)$ & Complanatuside isomer & - & + \\
\hline B4 & 5.23 & $\mathrm{~N}$ & $\mathrm{C}_{28} \mathrm{H}_{31} \mathrm{O}_{16}$ & 623.16231 & 623.16364 & 4.780 & $\mathrm{MS}^{2}[623]: 299(100), 284(32), 443(10), 240(4), 461(3), 577(2), 605(2), 211(2), 239(2)$ & Complanatuside isomer & - & + \\
\hline \multirow[t]{2}{*}{ B5 } & \multirow[t]{2}{*}{5.35} & $\mathrm{~N}$ & $\mathrm{C}_{22} \mathrm{H}_{21} \mathrm{O}_{11}$ & 461.10948 & 461.11050 & 3.773 & $\begin{array}{c}\operatorname{MS}^{2}[461]: 299(100), 284(9) \\
\operatorname{MS}^{3}[299]: 284(100)\end{array}$ & \multirow[t]{2}{*}{$\begin{array}{l}\text { Kaempferol-4'-methylether- } \\
\text { 3-D-glucoside }\end{array}$} & \multirow[t]{2}{*}{+} & \multirow[t]{2}{*}{ ++ } \\
\hline & & $\mathrm{P}$ & $\mathrm{C}_{22} \mathrm{H}_{23} \mathrm{O}_{11}$ & 463.12404 & 463.12265 & -1.809 & $\mathrm{MS}^{2}[463]: 445(100), 401(4), 344(4), 234(3), 431(1), 301(1)$ & & & \\
\hline B6 & 5.53 & $\mathrm{P}$ & $\mathrm{C}_{16} \mathrm{H}_{17} \mathrm{O}_{5}$ & 289.10760 & 289.10645 & -2.076 & $\mathrm{MS}^{2}[289]: 271(100), 270(91), 221(78), 205(76), 233(32), 261(17)$ & $\begin{array}{l}\text { (3R)-7, }, 2^{\prime}, 3^{\prime} \text {-trihydroxy-4'- } \\
\text { methoxy isoflavonone/isomer }\end{array}$ & + & - \\
\hline \multirow[t]{2}{*}{ B7 } & \multirow[t]{2}{*}{5.99} & $\mathrm{~N}$ & $\mathrm{C}_{16} \mathrm{H}_{11} \mathrm{O}_{5}$ & 283.06175 & 283.06198 & 3.642 & $\begin{array}{l}\operatorname{MS}^{2}[283]: 268(100), 269(3) \\
\operatorname{MS}^{3}[268]: 240(100), 239(49), 334(46), 211(44), 195(23)\end{array}$ & \multirow[t]{2}{*}{ Calycosin isomer } & \multirow[t]{2}{*}{ - } & \multirow[t]{2}{*}{+} \\
\hline & & $\mathrm{P}$ & $\mathrm{C}_{16} \mathrm{H}_{13} \mathrm{O}_{5}$ & 285.07630 & 285.07529 & -1.614 & $\mathrm{MS}^{2}[285]: 270(100), 253(44), 225(18), 137(7), 271(5), 257(3)$ & & & \\
\hline B8 & 6.19 & $\mathrm{~N}$ & $\mathrm{C}_{24} \mathrm{H}_{23} \mathrm{O}_{12}$ & 503.12005 & 503.12112 & 4.401 & $\mathrm{MS}^{2}[503]: 299(100), 284(23), 443(4), 461(2), 484(1), 240(1)$ & Neocomplanoside/isomer & - & + \\
\hline \multirow[t]{2}{*}{ B9* } & \multirow[t]{2}{*}{6.20} & $\mathrm{~N}$ & $\mathrm{C}_{22} \mathrm{H}_{21} \mathrm{O}_{10}$ & 445.11457 & 445.11481 & 4.239 & $\begin{array}{l}\mathrm{MS}^{2}[445]: 283(100), 268(17) \\
\text { MS }^{2}[4471: 285(100): 334(8), 403(2) 306(1) 241(1)\end{array}$ & \multirow[t]{2}{*}{ Calycosin-7-glucoside } & \multirow[t]{2}{*}{+} & \multirow[t]{2}{*}{++} \\
\hline & & $\mathrm{P}$ & $\mathrm{C}_{22} \mathrm{H}_{23} \mathrm{O}_{10}$ & 447.12912 & 447.12695 & -3.630 & $\begin{array}{c}\operatorname{MS}^{2}[4477: 285(1000,334(8), 403(2), 306(1), 241(1) \\
\operatorname{MS}^{3}[285]: 270(100), 253(41), 225(17), 137(7), 229(5), 211(5)\end{array}$ & & & \\
\hline B10 & 6.26 & $\mathrm{P}$ & $\mathrm{C}_{16} \mathrm{H}_{13} \mathrm{O}_{5}$ & 285.07630 & 285.07678 & 3.613 & $\begin{array}{c}\operatorname{MS}^{2}[285]: 270(100), 253(43), 225(19), 137(9) \\
\operatorname{MS}^{3}[270]: 137(100), 253(57), 214(31), 242(13), 134(12), 213(12)\end{array}$ & Calycosin isomer & - & + \\
\hline B11 & 6.34 & $\mathrm{P}$ & $\mathrm{C}_{17} \mathrm{H}_{15} \mathrm{O}_{6}$ & 315.08686 & 315.08603 & -0.903 & $\mathrm{MS}^{2}[315]: 300(100), 283(20), 255(8), 167(5), 259(4), 301(2), 287(2), 175(2)$ & $\begin{array}{l}\text { 7,3'-dihydroxy-8,4- } \\
\text { dimethoxyisoflavone/isomer }\end{array}$ & - & + \\
\hline B12 & 6.22 & $\mathrm{~N}$ & $\mathrm{C}_{22} \mathrm{H}_{21} \mathrm{O}_{12}$ & 477.10440 & 477.10532 & 4.382 & $\mathrm{MS}^{2}[477]: 315(100), 301(18), 300(14), 347(13), 313(11), 458(5), 278(4)$ & isorhamnetin-3-D- glucoside & + & ++ \\
\hline B13 & 6.35 & $\mathrm{~N}$ & $\mathrm{C}_{17} \mathrm{H}_{13} \mathrm{O}_{6}$ & 313.07231 & 313.07236 & 4.415 & $\mathrm{MS}^{2}[313]: 298(100), 285(2), 295(1), 287(1), 283(1)$ & $\begin{array}{c}7,3^{\prime} \text {-dihydroxy-8,4- } \\
\text { dimethoxvisoflavone/isomer }\end{array}$ & + & ++ \\
\hline B14 & 6.49 & $\mathrm{~N}$ & $\mathrm{C}_{22} \mathrm{H}_{21} \mathrm{O}_{9}$ & 429.11965 & 429.12024 & 4.200 & $\mathrm{MS}^{2}[429]: 252(100), 253(25), 295(7), 267(3), 411(2), 361(2), 383(2), 231(2)$ & Ononin isomer & + & - \\
\hline \multirow[t]{2}{*}{ B17 } & \multirow[t]{2}{*}{6.82} & $\mathrm{~N}$ & $\mathrm{C}_{23} \mathrm{H}_{23} \mathrm{O}_{11}$ & 475.12513 & 475.12619 & 3.687 & $\begin{array}{c}\operatorname{MS}^{2}[475]: 299(100), 284(13), 298(7), 460(5), 283(2), 297(2), 431(1) \\
\operatorname{MS}^{3}[299]: 284(100), 240(1)\end{array}$ & \multirow{3}{*}{$\begin{array}{l}\text { Odoratin-7-O- } \beta \text {-D- } \\
\text { glucoside/isomer }\end{array}$} & \multirow[t]{2}{*}{+} & \multirow[t]{2}{*}{ - } \\
\hline & & $\mathrm{P}$ & $\mathrm{C}_{23} \mathrm{H}_{25} \mathrm{O}_{11}$ & 477.13969 & 477.13742 & -3.601 & $\begin{array}{c}\mathrm{MS}^{2}[477]: 301(100), 345(10), 199(10), 183(8), 453(7) \\
\mathrm{MS}^{3}[301]: 286(100), 269(33), 153(29), 245(15), 241(14), 152(6), 223(5), 175(2), 273(2), 123(1)\end{array}$ & & & \\
\hline \multirow{2}{*}{ B18 } & \multirow{2}{*}{6.99} & $\mathrm{~N}$ & $\mathrm{C}_{16} \mathrm{H}_{11} \mathrm{O}_{5}$ & 283.06175 & 283.06192 & 4.430 & & & \multirow{2}{*}{++} & \multirow{2}{*}{+} \\
\hline & & $\mathrm{P}$ & $\mathrm{C}_{16} \mathrm{H}_{13} \mathrm{O}_{5}$ & 285.07630 & 285.07529 & -1.614 & $\begin{array}{c}\operatorname{MS}^{3}[268]: 240(100), 239(63), 211(55), 224(40), 184(28), 195(27) \\
\text { MS }^{2}[285]: 270(100), 253(43), 225(20), 285(17), 137(9), 229(7), 286(4), 257(3), 181(2)\end{array}$ & Calycosin isomer & & \\
\hline B19 & 7.02 & $\begin{array}{l}\mathrm{N} \\
\mathrm{P}\end{array}$ & $\begin{array}{l}\mathrm{C}_{16} \mathrm{H}_{15} \mathrm{O}_{5} \\
\mathrm{C}_{16} \mathrm{H}_{17} \mathrm{O}_{5}\end{array}$ & $\begin{array}{l}287.09305 \\
289.10760\end{array}$ & $\begin{array}{l}287.09225 \\
289.10651\end{array}$ & $\begin{array}{c}2.961 \\
-1.868\end{array}$ & $\begin{array}{c}\mathrm{MS}^{2}[287]: 243(100), 203(53), 201(19), 219(11), 259(9), 173(7), 157(5) \\
\operatorname{MS}^{2}[289]: 271(100), 184(8), 252(8), 166(7), 205(4), 182(2)\end{array}$ & $\begin{array}{l}\text { (3R)-7, }, 2^{\prime}, 3^{\prime} \text {-trihydroxy-4'- } \\
\text { methoxy isoflavonone/isomer }\end{array}$ & + & - \\
\hline B20 & 7.10 & $\mathrm{~N}$ & $\mathrm{C}_{22} \mathrm{H}_{21} \mathrm{O}_{10}$ & 445.11457 & 445.11575 & 4.351 & $\begin{array}{l}\mathrm{MS}^{2}[445]: 283(100), 268(17) \\
\quad \mathrm{MS}^{3}[283]: 268(100)\end{array}$ & Calycosin-7-glucoside isomer & - & + \\
\hline B21 & 7.16 & $\mathrm{~N}$ & $\mathrm{C}_{17} \mathrm{H}_{13} \mathrm{O}_{6}$ & 313.07231 & 313.07230 & 3.224 & $\mathrm{MS}^{2}[313]: 298(100), 181(17), 245(8), 137(6), 295(6), 269(5), 139(5), 131(3), 194(3)$ & $\begin{array}{l}\text { 7,3'-dihydroxy-8,4- } \\
\text { dimethoxyisoflavone/isomer }\end{array}$ & + & ++ \\
\hline B22 & 7.25 & $\mathrm{~N}$ & $\mathrm{C}_{21} \mathrm{H}_{19} \mathrm{O}_{10}$ & 431.09892 & 431.09961 & 3.421 & $\mathrm{MS}^{2}[431]: 268(100), 269(48), 311(8), 413(6), 341(4), 323(2), 412(2)$ & genistin isomer & - & + \\
\hline B23 & 7.28 & $\mathrm{P}$ & $\mathrm{C}_{17} \mathrm{H}_{15} \mathrm{O}_{6}$ & 315.08686 & 315.08575 & -1.792 & $\mathrm{MS}^{2}[315]: 300(100), 283(19), 255(9), 269(8), 297(5), 167(5), 259(4), 138(3)$ & $\begin{array}{l}\text { 7,3'-dihydroxy-8,4- } \\
\text { dimethoxyisoflavone/isomer }\end{array}$ & + & - \\
\hline B24 & 7.36 & $\mathrm{~N}$ & $\mathrm{C}_{15} \mathrm{H}_{9} \mathrm{O}_{5}$ & 269.04610 & 269.04352 & -3.456 & $\mathrm{MS}^{2}[269]: 241(100), 240(58), 225(48), 197(25), 185(20), 213(15)$ & $\begin{array}{l}5,7,4^{\prime} \text {-trihydroxy- } \\
\text { isoflavonone/isomer }\end{array}$ & - & + \\
\hline B25 & 7.38 & $\mathrm{~N}$ & $\mathrm{C}_{16} \mathrm{H}_{11} \mathrm{O}_{5}$ & 283.06175 & 283.06189 & 4.324 & $\begin{array}{c}\operatorname{MS}^{2}[283]: 268(100), 269(1) \\
\operatorname{MS}^{3}[268]: 240(100), 211(58), 239(55), 224(40), 195(25)\end{array}$ & Calycosin isomer & + & ++ \\
\hline & & $\mathrm{P}$ & $\mathrm{C}_{16} \mathrm{H}_{13} \mathrm{O}_{5}$ & 285.07630 & 285.07571 & -0.140 & $\begin{array}{l}\mathrm{MS}^{2}[285]: 270(100), 253(43), 225(20), 285(14), 137(8), 229(7), 286(5), 257(3), 181(2), 197(1) \\
\left.\mathrm{MS}^{3}, 270\right]: 137(100), 253(54), 214(33), 23(14), 134(13) 242(12)\end{array}$ & & & \\
\hline B26* & 7.52 & $\mathrm{~N}$ & $\mathrm{C}_{28} \mathrm{H}_{31} \mathrm{O}_{16}$ & 623.16231 & 623.16339 & 4.379 & $\mathrm{MS}^{2}[623]: 461(100), 299(68), 443(3)$ & Complanaruside & - & + \\
\hline
\end{tabular}


Table 2. Cont.

\begin{tabular}{|c|c|c|c|c|c|c|c|c|c|c|}
\hline Peak & $\begin{array}{c}t_{R} \\
/ \mathrm{min}\end{array}$ & Ion mode & Formula & $\begin{array}{l}\text { Theoretical } \\
\text { Mass } m / z\end{array}$ & $\begin{array}{l}\text { Experimental } \\
\text { Mass } m / z\end{array}$ & Error (ppm) & $\mathrm{MS}^{2} / \mathrm{MS}^{3}$ fragment ions & Identification & FAR & AR \\
\hline B27 & 7.56 & $\mathrm{~N}$ & $\mathrm{C}_{16} \mathrm{H}_{11} \mathrm{O}_{5}$ & 283.06175 & 283.06158 & 3.229 & MS $^{2}[283]: 268(100), 254(7), 269(3), 253(1), 255(1), 239(1), 265(1)$ & Calycosin isomer & + & - \\
\hline B28 & 7.69 & $\mathrm{P}$ & $\mathrm{C}_{16} \mathrm{H}_{13} \mathrm{O}_{5}$ & 285.07630 & 285.07550 & -0.877 & $\begin{array}{l}\operatorname{MS}^{2}[285]: 270(100), 253(43), 225(19), 137(9), 229(7), 257(3), 181(2) \\
\operatorname{MS}^{3}[270]: 137(100), 253(58), 214(29), 213(15), 134(11), 242(11)\end{array}$ & Calycosin isomer & + & - \\
\hline B29 & 7.70 & $\mathrm{~N}$ & $\mathrm{C}_{24} \mathrm{H}_{23} \mathrm{O}_{11}$ & 487.12513 & 487.12631 & 3.793 & $\mathrm{MS}^{2}[487]: 283(100), 268(50), 427(14), 193(11), 419(10), 253(3)$ & $\begin{array}{l}\text { Calycosin-7-O- } \beta \text {-D-glucoside- } \\
6 \text { "-o-acetate }\end{array}$ & - & + \\
\hline B30 & 7.80 & $\mathrm{P}$ & $\mathrm{C}_{17} \mathrm{H}_{15} \mathrm{O}_{6}$ & 315.08686 & 315.08594 & -1.189 & $\mathrm{MS}^{2}[315]: 300(100), 283(19), 255(7), 167(5), 301(5), 259(4), 138(3), 269(3), 168(2), 297(1)$ & $\begin{array}{l}\text { 7,3'-dihydroxy-8,4- } \\
\text { dimethoxyisoflavone/isomer }\end{array}$ & + & ++ \\
\hline B31 & 7.87 & $\begin{array}{l}\mathrm{N} \\
\mathrm{P}\end{array}$ & $\begin{array}{l}\mathrm{C}_{16} \mathrm{H}_{11} \mathrm{O}_{4} \\
\mathrm{C}_{16} \mathrm{H}_{13} \mathrm{O}_{4}\end{array}$ & $\begin{array}{l}267.06683 \\
269.08138\end{array}$ & $\begin{array}{l}267.06693 \\
269.08215\end{array}$ & $\begin{array}{l}4.533 \\
4.886\end{array}$ & $\begin{array}{c}\mathrm{MS}^{2}[267]: 252(100), 253(5), 249(2) \\
\operatorname{MS}^{2}[269]: 254(100), 237(51), 213(35), 253(13), 107(9), 118(6), 241(6), 136(5)\end{array}$ & Formononetin isomer & + & ++ \\
\hline B32 & 7.89 & $\mathrm{P}$ & $\mathrm{C}_{26} \mathrm{H}_{27} \mathrm{O}_{11}$ & 515.15534 & 515.15131 & -3.751 & $\mathrm{MS}^{2}[515]: 500(100), 485(76), 339(75), 484(56), 338(31), 324(30), 497(27), 337(19), 323(18)$ & $\begin{array}{l}\text { Calycosin-7-O- } \beta \text {-D-glucoside- } \\
6^{\prime \prime}-\text { o-butylene ester/isomer }\end{array}$ & + & - \\
\hline B33 & 7.93 & $\mathrm{~N}$ & $\mathrm{C}_{29} \mathrm{H}_{37} \mathrm{O}_{15}$ & 625.21434 & 625.21527 & 4.116 & $\mathrm{MS}^{2}[625]: 301(100), 463(9), 286(4), 445(3), 607(2), 271(2), 473(1)$ & $\begin{array}{l}\text { Isomucronulatol-1-7,2'- } \\
\text { di-o-glucoside/isomer }\end{array}$ & + & ++ \\
\hline В34 & 8.12 & $\mathrm{~N}$ & $\mathrm{C}_{16} \mathrm{H}_{11} \mathrm{O}_{5}$ & 283.06175 & 283.06168 & 3.582 & $\operatorname{MS}^{2}[283]: 268(100), 269(3), 255(1)$ & Calycosin isomer & - & + \\
\hline B35 & 8.15 & $\mathrm{~N}$ & $\mathrm{C}_{23} \mathrm{H}_{23} \mathrm{O}_{11}$ & 475.12513 & 475.12598 & 3.245 & $\mathrm{MS}^{2}[475]: 298(100), 297(48), 299(30), 283(23), 269(12), 284(10), 457(10), 277(6)$ & $\begin{array}{l}\text { 7,3'-dihydroxy-8,4- } \\
\text { dimethoxyisoflavone/isomer }\end{array}$ & + & - \\
\hline B36 & 8.24 & $\mathrm{~N}$ & $\mathrm{C}_{16} \mathrm{H}_{11} \mathrm{O}_{4}$ & 267.06683 & 267.06702 & 4.870 & $\begin{array}{c}\operatorname{MS}^{2}[267]: 252(100), 253(1) \\
\operatorname{MS}^{3}[252]: 223(100), 208(65), 224(54), 132(21), 195(15), 196(5)\end{array}$ & Formononetin isomer & + & ++ \\
\hline B37* & 8.26 & $\mathrm{P}$ & $\mathrm{C}_{22} \mathrm{H}_{23} \mathrm{O}_{9}$ & 431.13421 & 431.13263 & -2.386 & $\mathrm{MS}^{2}[431]: 269(100), 343(0.3), 413(0.2)$ & Ononin & - & + \\
\hline B38 & 8.41 & $\begin{array}{l}\mathrm{N} \\
\mathrm{P}\end{array}$ & $\begin{array}{l}\mathrm{C}_{17} \mathrm{H}_{15} \mathrm{O}_{5} \\
\mathrm{C}_{17} \mathrm{H}_{17} \mathrm{O}_{5}\end{array}$ & $\begin{array}{r}299.09305 \\
301.10760\end{array}$ & $\begin{array}{l}299.09293 \\
301.10641\end{array}$ & $\begin{array}{c}3.115 \\
-2.126\end{array}$ & $\begin{array}{c}\operatorname{MS}^{2}[299]: 284(100), 269(1), 255(1) \\
\operatorname{MS}^{2}[301]: 167(100), 269(26), 191(21), 147(19), 163(12), 273(11), 207(9), 286(6), 241(6), 270(3)\end{array}$ & Pratensein/isomer & ++ & + \\
\hline B39 & 8.53 & $\mathrm{P}$ & $\mathrm{C}_{26} \mathrm{H}_{27} \mathrm{O}_{11}$ & 515.15534 & 515.15076 & -4.819 & $\mathrm{MS}^{2}[515]: 339(100), 321(3), 199(1)$ & $\begin{array}{l}\text { Calycosin-7-O- } \beta \text {-D- } \\
\text { glucoside-6"--O-butylene } \\
\text { ester }\end{array}$ & + & - \\
\hline B40 & 8.54 & $\mathrm{~N}$ & $\mathrm{C}_{16} \mathrm{H}_{15} \mathrm{O}_{5}$ & 287.09305 & 287.09183 & 1.498 & $\mathrm{MS}^{2}[287]: 272(100), 135(93), 165(46), 177(29), 121(22), 147(19)$ & $\begin{array}{l}\text { (3R)-7, } 2^{\prime}, 3^{\prime} \text {-trihydroxy-4'- } \\
\text { methoxy isoflavonone/isomer }\end{array}$ & - & + \\
\hline B42 & 8.73 & $\mathrm{~N}$ & $\mathrm{C}_{23} \mathrm{H}_{23} \mathrm{O}_{11}$ & 475.12513 & 475.12601 & 3.308 & $\mathrm{MS}^{2}[475]: 299(100), 284(62), 298(18), 297(17), 283(9), 285(9), 269(9), 151(1)$ & $\begin{array}{l}\text { Odoratin-7-O- } \beta \text {-D- } \\
\text { glucoside/isomer }\end{array}$ & + & - \\
\hline B43 & 8.78 & $\mathrm{~N}$ & $\mathrm{C}_{16} \mathrm{H}_{11} \mathrm{O}_{4}$ & 267.06683 & 267.06696 & 4.158 & $\operatorname{MS}^{2}[267]: 252(100), 253(3), 249(2), 223(1)$ & Formononetin isomer & - & + \\
\hline B44 & $\begin{array}{l}8.96 \\
9.00\end{array}$ & $\begin{array}{l}\mathrm{N} \\
\mathrm{P}\end{array}$ & $\begin{array}{l}\mathrm{C}_{17} \mathrm{H}_{15} \mathrm{O}_{5} \\
\mathrm{C}_{17} \mathrm{H}_{17} \mathrm{O}_{5}\end{array}$ & $\begin{array}{l}299.09305 \\
301.10760\end{array}$ & $\begin{array}{l}299.09314 \\
301.10641\end{array}$ & $\begin{array}{l}3.817 \\
-2.126\end{array}$ & $\begin{array}{c}\operatorname{MS}^{2}[299]: 284(100), 269(4) \\
\operatorname{MS}^{2}[301]: 167(100), 269(22), 191(20), 147(15), 163(10), 273(9), 207(7), 241(6), 286(2), 270(2)\end{array}$ & Pratensein/isomer & + & ${ }^{++}$ \\
\hline B45 & $\begin{array}{l}9.03 \\
9.08\end{array}$ & $\begin{array}{l}\mathrm{N} \\
\mathrm{P}\end{array}$ & $\begin{array}{l}\mathrm{C}_{17} \mathrm{H}_{17} \mathrm{O}_{5} \\
\mathrm{C}_{17} \mathrm{H}_{19} \mathrm{O}_{5}\end{array}$ & $\begin{array}{l}301.10870 \\
303.12325\end{array}$ & $\begin{array}{l}301.10770 \\
303.12225\end{array}$ & $\begin{array}{c}3.479 \\
-1.485\end{array}$ & $\begin{array}{l}\operatorname{MS}^{2}[301]: 286(100), 109(14), 135(12), 147(10), 283(8), 271(6), 179(3), 153(2), 257(2) \\
\text { SS }^{2}[303]: 167(100), 149(32), 123(19), 284(16), 181(14), 168(7), 219(6), 270(5), 193(5)\end{array}$ & $\begin{array}{l}\text { (3R)-8, } 2^{\prime} \text {-Dihydroxy-7,4'- } \\
\text { dimethoxy-isoflavan/isomer }\end{array}$ & ++ & + \\
\hline B46 & 9.17 & $\mathrm{~N}$ & $\mathrm{C}_{16} \mathrm{H}_{11} \mathrm{O}_{4}$ & 267.06683 & 267.06689 & 3.046 & $\begin{array}{c}\operatorname{MS}^{2}[267]: 252(100), 253(5) \\
\operatorname{MS}^{3}[252]: 223(100), 208(70), 224(46), 132(16), 195(15), 196(7), 179(3), 225(2)\end{array}$ & Formononetin isomer & + & - \\
\hline & & $\mathrm{P}$ & $\mathrm{C}_{16} \mathrm{H}_{13} \mathrm{O}_{4}$ & 269.08138 & 269.08182 & 3.659 & $\begin{array}{c}\operatorname{MS}^{2}[269]: 269(100), 254(72), 237(40), 213(29), 270(18), 253(10), 107(7), 118(4), 136(3) \\
\operatorname{MS}^{3}[269]: 254(100), 253(32), 214(11), 163(7)\end{array}$ & & & \\
\hline B47 & 9.23 & $\mathrm{~N}$ & $\mathrm{C}_{17} \mathrm{H}_{17} \mathrm{O}_{5}$ & 301.10870 & 301.10880 & 3.811 & 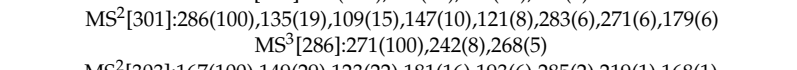 & $\begin{array}{l}\text { (3R)-8, } 2^{\prime} \text {-dihydroxy-7, } 4^{\prime}- \\
\text { dimethoxy-isoflavan/isomer }\end{array}$ & - & + \\
\hline & 9.24 & $\mathrm{P}$ & $\mathrm{C}_{17} \mathrm{H}_{19} \mathrm{O}_{5}$ & 303.12325 & 303.12219 & -1.683 & $\begin{array}{c}\operatorname{MS}^{2}[303]: 167(100), 149(29), 123(22), 181(16), 193(6), 285(2), 219(1), 168(1) \\
\operatorname{MS}^{2}[463]: 301(100), 299(1)\end{array}$ & Astraisoflavan-7-O- $\beta$-D- & & \\
\hline B48* & 9.25 & N & $\mathrm{C}_{23} \mathrm{H}_{27} \mathrm{O}_{10}$ & 463.16152 & 463.16254 & 3.757 & $\begin{array}{l}\mathrm{MS}^{2}[463][301(100), 299(1) \\
\quad \mathrm{MS}^{3}[301]: 286(100)\end{array}$ & glucoside & + & ${ }^{++}$ \\
\hline B49 & 9.40 & $\begin{array}{l}\mathrm{N} \\
\mathrm{P}\end{array}$ & $\begin{array}{l}\mathrm{C}_{17} \mathrm{H}_{13} \mathrm{O}_{5} \\
\mathrm{C}_{17} \mathrm{H}_{15} \mathrm{O}_{5}\end{array}$ & $\begin{array}{l}297.07740 \\
299.09195\end{array}$ & $\begin{array}{l}297.07748 \\
299.09119\end{array}$ & $\begin{array}{c}3.823 \\
-0.702\end{array}$ & $\begin{array}{c}\mathrm{MS}^{2}[297]: 282(100), 283(4), 279(3), 267(2), 253(2), 254(1), 167(1) \\
\mathrm{MS}^{2}[299]: 284(100), 166(23), 243(21), 239(11), 267(11), 285(10), 137(4) \\
\mathrm{MS}^{3}[284]: 256(100), 267(27), 166(16), 253(10), 255(8), 227(8), 254(6), 241(5)\end{array}$ & Afromosin & ++ & + \\
\hline B50 & 9.42 & N & $\mathrm{C}_{23} \mathrm{H}_{27} \mathrm{O}_{10}$ & 463.16152 & 463.16241 & 3.477 & $\operatorname{MS}^{2}[463]: 287(100), 272(3), 395(3), 213(1)$ & $\begin{array}{l}\text { Astraisoflavan-7-O- } \beta \text {-D- } \\
\text { glucoside isomer }\end{array}$ & + & - \\
\hline
\end{tabular}


Table 2. Cont

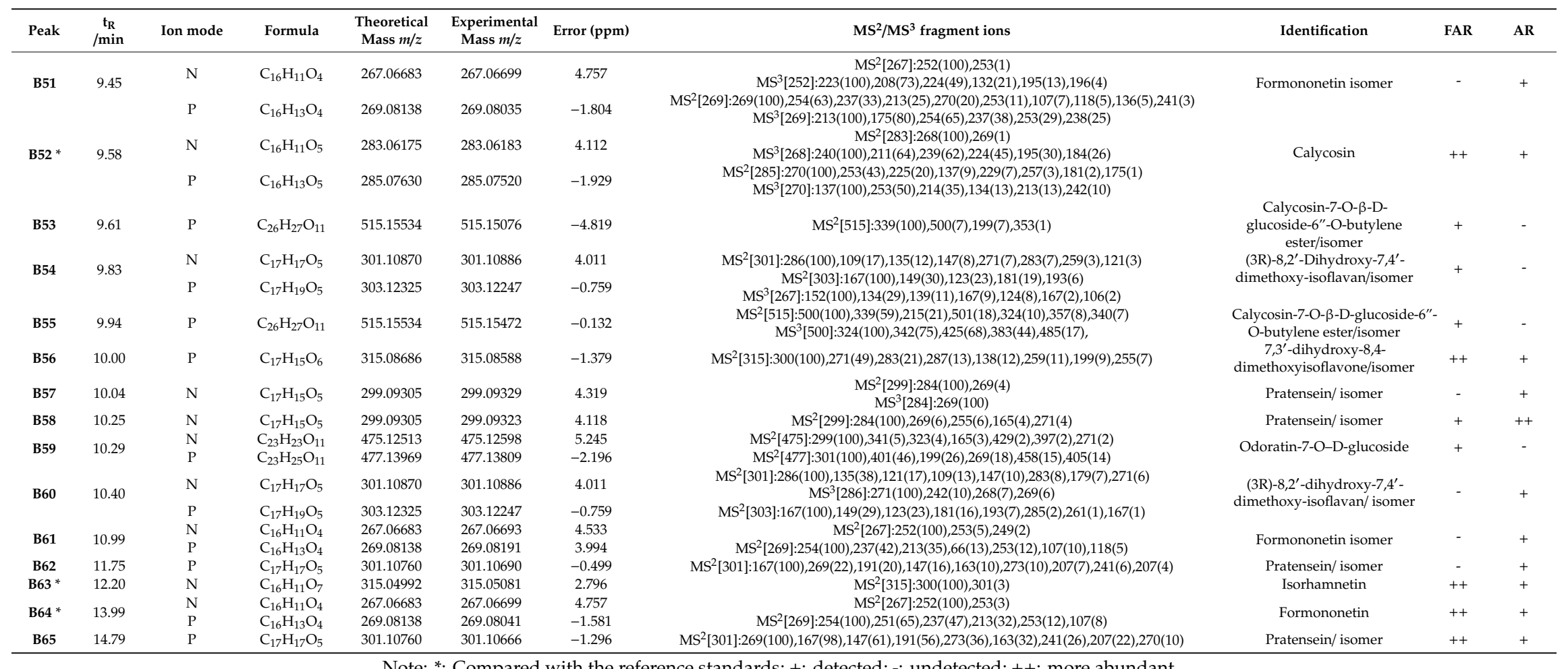

Note: *: Compared with the reference standards; +: detected; -: undetected; ++: more abundant. 

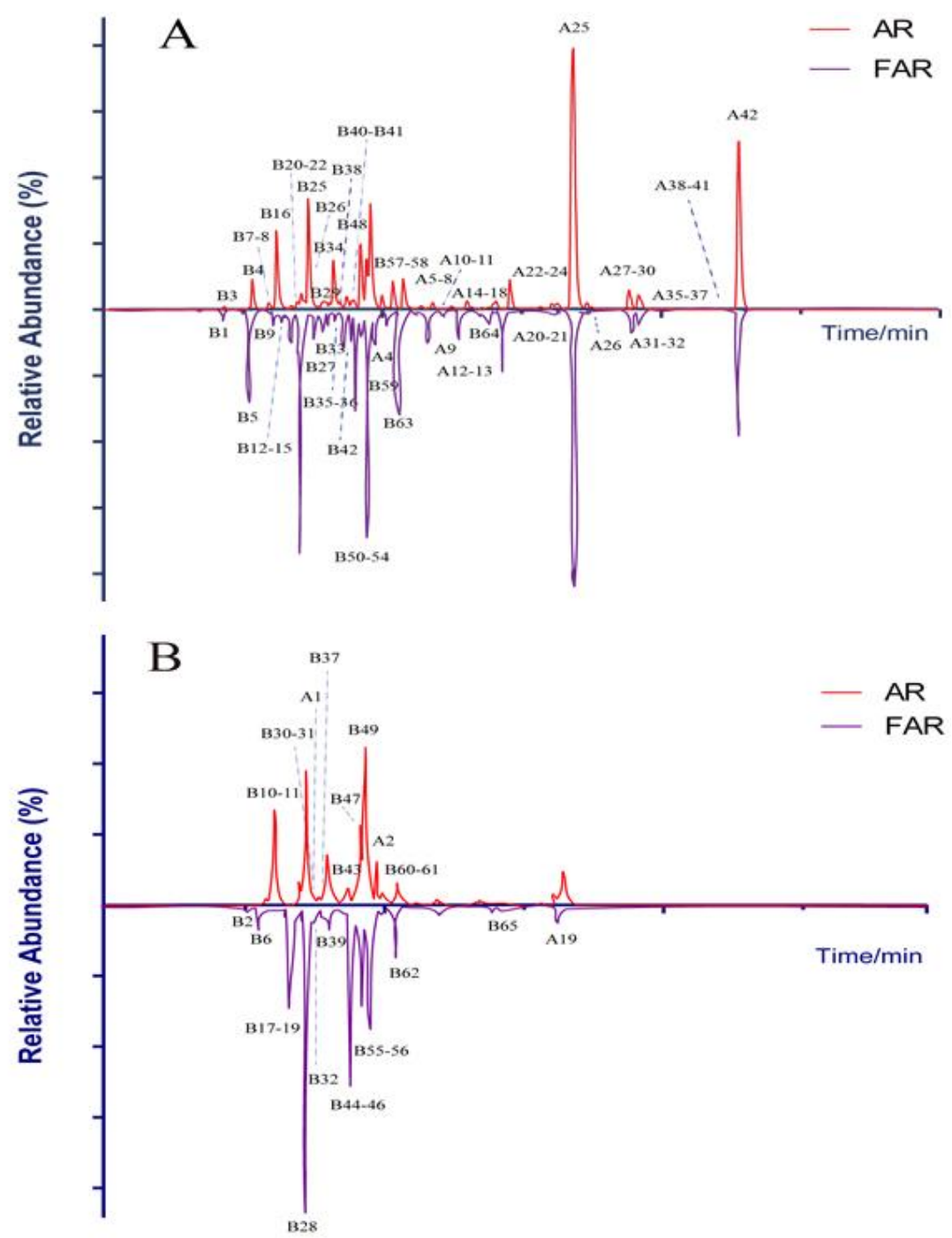

Figure 2. High-resolution extracted ion chromatograms for 107 compounds in AR and FAR. (A) Results of negative ion mode; $(\mathbf{B})$ results of positive ion mode.

\subsubsection{Structural Assignment of Saponins in AR and FAR}

Most of the saponins in AR possess the same aglycone of cycloastragenol with different substituent groups, such as xylose, glucose, acetyl groups, and so on. They can be divided into type cyclolanostane cycloastragenol (1-11) or cyclolanostane cyclocanthogenin (12-18). Only a minority of saponins belonged to oleanane-type triterpenoids (19), the aglycones of which are attributed to soybean saponin B. There were 29 and 42 saponins screened and identified in FAR and AR, respectively, and their molecular formulae and chemical structures are shown in Table 3. 
Table 3. Chemical information of identified saponins in AR and FAR.

\begin{tabular}{|c|c|c|c|c|}
\hline No & Name & Formula & Core Structure & Substituent Group \\
\hline 1 & Astragaloside I & $\mathrm{C}_{45} \mathrm{H}_{72} \mathrm{O}_{16}$ & & $\mathrm{R}_{1}=$ glu $\mathrm{R}_{2}=\mathrm{R}_{5}=\mathrm{H} \mathrm{R}_{3}=\mathrm{R}_{4}=\mathrm{Ac}$ \\
\hline 2 & Isoastragaloside I & $\mathrm{C}_{45} \mathrm{H}_{72} \mathrm{O}_{16}$ & & $\mathrm{R}_{1}=\mathrm{glu} \mathrm{R}_{2}=\mathrm{R}_{4}=\mathrm{H} \mathrm{R}_{3}=\mathrm{R}_{5}=\mathrm{Ac}$ \\
\hline 3 & Astragaloside II & $\mathrm{C}_{43} \mathrm{H}_{70} \mathrm{O}_{15}$ & & $\mathrm{R}_{1}=$ glu $\mathrm{R}_{2}=\mathrm{R}_{4}=\mathrm{R}_{5}=\mathrm{H} \mathrm{R}_{3}=\mathrm{Ac}$ \\
\hline 4 & Isoastragaloside II & $\mathrm{C}_{43} \mathrm{H}_{70} \mathrm{O}_{15}$ & & $\mathrm{R}_{1}=$ glu $\mathrm{R}_{2}=\mathrm{R}_{3}=\mathrm{R}_{5}=\mathrm{H} \mathrm{R} \mathrm{R}_{4}=\mathrm{Ac}$ \\
\hline 5 & Astragaloside III & $\mathrm{C}_{41} \mathrm{H}_{68} \mathrm{O}_{14}$ & & $\mathrm{R}_{1}=\mathrm{R}_{2}=\mathrm{glu} \mathrm{R}_{5}=\mathrm{H} \mathrm{R}_{3}=\mathrm{R}_{4}=\mathrm{Ac}$ \\
\hline 6 & Astragaloside IV & $\mathrm{C}_{41} \mathrm{H}_{68} \mathrm{O}_{14}$ & & $\mathrm{R}_{1}=$ glu $\mathrm{R}_{2}=\mathrm{R}_{3}=\mathrm{R}_{4}=\mathrm{R}_{5}=\mathrm{H}$ \\
\hline 7 & Isoastragaloside IV & $\mathrm{C}_{41} \mathrm{H}_{68} \mathrm{O}_{14}$ & & $\mathrm{R}_{1}=\mathrm{R}_{3}=\mathrm{R}_{4}=\mathrm{R}_{5}=\mathrm{H} \mathrm{R} \mathrm{R}_{2}=\mathrm{glu}$ \\
\hline 8 & Acetylastragaloside I & $\mathrm{C}_{47} \mathrm{H}_{74} \mathrm{O}_{17}$ & & $\mathrm{R}_{1}=$ glu $\mathrm{R}_{2}=\mathrm{H} \mathrm{R}_{3}=\mathrm{R}_{4}=\mathrm{R}_{5}=\mathrm{Ac}$ \\
\hline 9 & Agroastragaloside III & $\mathrm{C}_{51} \mathrm{H}_{82} \mathrm{O}_{21}$ & & $\mathrm{R}_{1}=\mathrm{R}_{2}=\mathrm{glu} \mathrm{R}_{5}=\mathrm{H} \mathrm{R}_{3}=\mathrm{R}_{4}=\mathrm{Ac}$ \\
\hline 10 & Malonylastragaloside I & $\mathrm{C}_{48} \mathrm{H}_{74} \mathrm{O}_{19}$ & & $\begin{array}{c}\mathrm{R}_{1}=\mathrm{glu} \mathrm{R}_{2}=\mathrm{H} \mathrm{R}_{3}=\mathrm{R}_{4}=\mathrm{Ac} \\
\mathrm{R}_{5}=\text { malonyl }\end{array}$ \\
\hline 11 & Astramembranoside A & $\mathrm{C}_{42} \mathrm{H}_{70} \mathrm{O}_{15}$ & & $\begin{array}{c}\mathrm{R}_{1}=\mathrm{H} \mathrm{R}_{2}=\alpha-\mathrm{O}-\mathrm{glu} \beta-\mathrm{H} \\
\mathrm{R}_{3}=\mathrm{glu}\end{array}$ \\
\hline
\end{tabular}

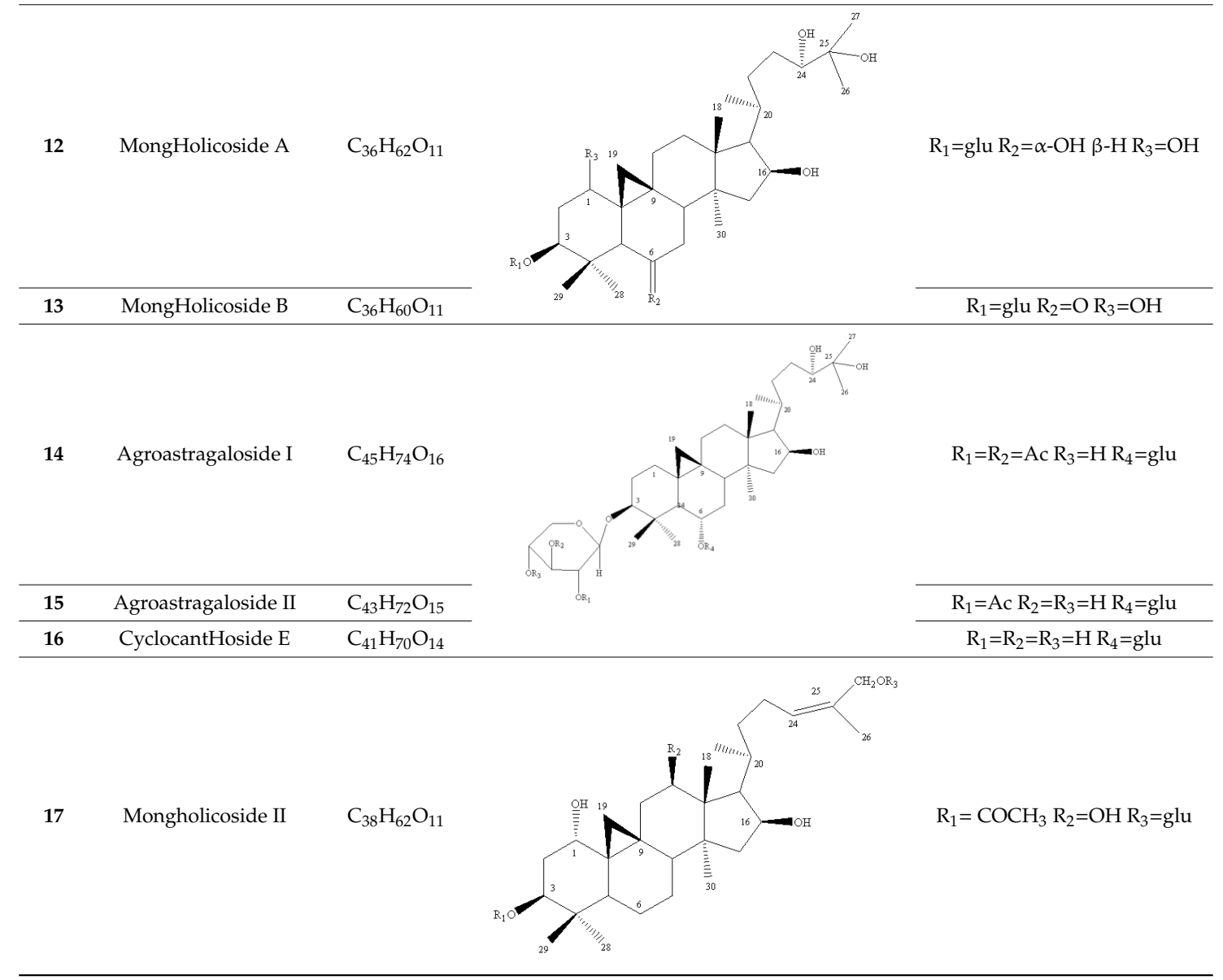


Table 3. Cont.

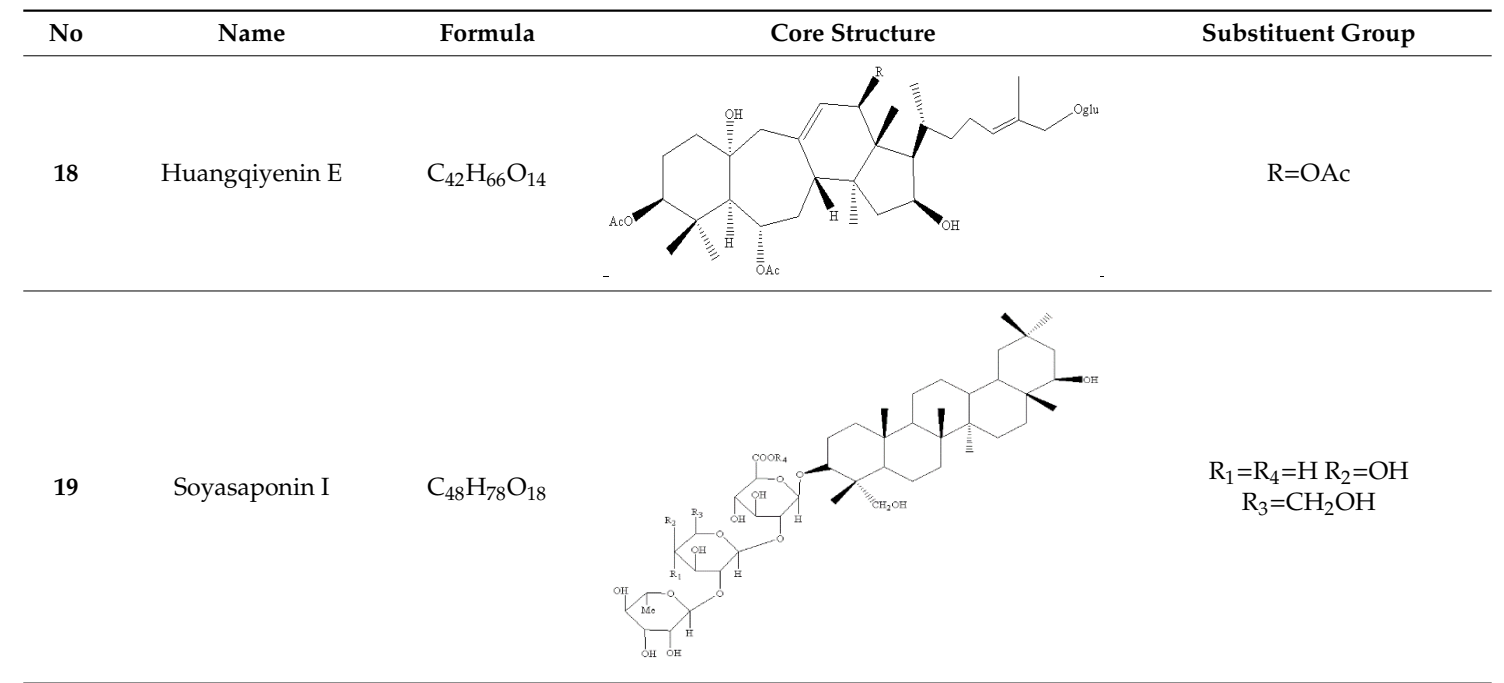

With retention times of 11.79 and $14.27 \mathrm{~min}, \mathbf{A 6}$ and $\mathbf{A 1 6}$ afforded $[\mathrm{M}-\mathrm{H}]^{-}$ions at $m / z 783.45612$ and $783.45813\left(\mathrm{C}_{41} \mathrm{H}_{67} \mathrm{O}_{14}\right.$, mass error within $\left.5 \mathrm{ppm}\right)$ in negative ion mode. Both of them produced the base peak ions at $m / z 489$ by neutral loss of the glucose and xylose moiety. Then, the product ion at $m / z$ 489 further generated the predominant ion at $m / z 453$ by loss of $2 \mathrm{H}_{2} \mathrm{O}$. Meanwhile, several important fragment ions at $m / z 651$ and $m / z 621$ were also observed due to the respective losses of xylose and glucose. Combined with standard substances, A6 was positively characterized as isoastragaloside IV, while A16 was speculated to be astragaloside IV.

A18 produced its $[\mathrm{M}-\mathrm{H}]^{-}$ion at $m / z 783.45654\left(\mathrm{C}_{41} \mathrm{H}_{67} \mathrm{O}_{14}\right)$ with a mass error of $3.11 \mathrm{ppm}$. In the ESI-MS ${ }^{2}$ spectrum, further mass fragmentation resulted in $m / z 489$ [M-H-Glu-Xyl] ${ }^{-}, m / z 621$ [M-H-Glu] $]^{-}$, and $m / z 453$ [M-H-Xyl-Glu- $2 \mathrm{H}_{2} \mathrm{O}^{-}$, consistent with the characteristic fragmentation pathways of astragalus saponins. By comparing with the reference standard, A18 was unambiguously identified as astragaloside III.

A2, A11, A14, A22, A25, and A27, which possessed a theoretical $[\mathrm{M}-\mathrm{H}]^{-}$ion at $\mathrm{m} / \mathrm{z} 825.46419$ $\left(\mathrm{C}_{43} \mathrm{H}_{69} \mathrm{O}_{15}\right.$, mass error within $\left.5 \mathrm{ppm}\right)$, were eluted at 9.32, 12.59, 14.00, 16.67, 16.91, and $18.12 \mathrm{~min}$, in order. In their ESI-MS ${ }^{2}$ spectra, the $[\mathrm{M}-\mathrm{H}]^{-}$ion at $\mathrm{m} / \mathrm{z} 825$ generated product ions at $\mathrm{m} / \mathrm{z} 783, \mathrm{~m} / \mathrm{z}$ 765 , and $m / z 633$ by losing acetyl, acetyl $+\mathrm{H}_{2} \mathrm{O}$, and xylose moieties. Among them, A22 was positively identified as astragaloside II, and A27 was unambiguously characterized as isoastragaloside II based on comparison of the MS/MS spectra and retention times with reference standards. The accurate mass weight and major product ions of A2, A11, A14, and A25 were coincident with those of A22, indicating that they could be astragaloside II isomers.

A30, A33, A36, A37, and A39 generated an identical $[\mathrm{M}-\mathrm{H}]^{-}$ion at $m / z 867.47476\left(\mathrm{C}_{45} \mathrm{H}_{71} \mathrm{O}_{16}\right)$ with mass errors within $5 \mathrm{ppm}$. All of their deprotonated molecular ions generated a series of fragment ions at $m / z$ 807, $m / z 765$, and $m / z$ 747, corresponding to [M-H-Ac- $\left.\mathrm{H}_{2} \mathrm{O}\right]^{-},\left[\mathrm{M}-\mathrm{H}-2 \mathrm{Ac}_{-} \mathrm{H}_{2} \mathrm{O}\right]^{-}$, and $\left[\mathrm{M}-\mathrm{H}-2 \mathrm{Ac}-2 \mathrm{H}_{2} \mathrm{O}\right]^{-}$. With the supplements of standard substances, A37 was unambiguously characterized as astragaloside I, while $\mathbf{A} \mathbf{3 9}$ was positively identified as isoastragaloside I. Therefore, A30, A33, and A36 were determined to be astragaloside I isomers.

The ESI-MS ${ }^{n}$ spectra of A16, A18, A22, and A37 are shown in Figure 3. 

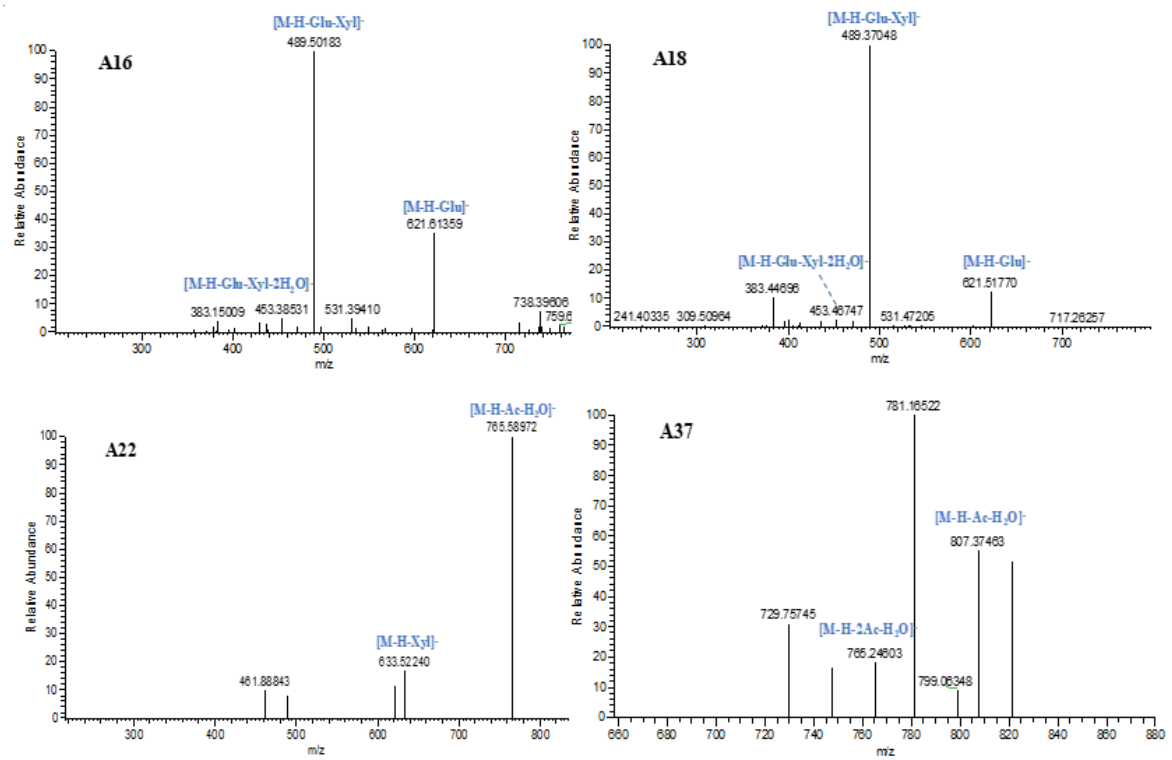

Figure 3. The ESI-MS ${ }^{\mathrm{n}}$ spectra of A16, A18, A22, and A37.

\subsubsection{Structural Assignment of Flavonoids in AR}

AR contains a large number of flavonoids and glycosides, which can be divided into flavones (1-5), isoflavans (6-10), and isoflavones (11-21). Among these, the isoflavones are the most important group. Their molecular formulae and chemical structures are summarized in Table 4 . In this work, 43 flavonoids in FAR and 47 flavonoids in AR were detected and characterized.

B9 and B20 possessed [M-H] $]^{-}$ions at $m / z 445.11481$ and $m / z 445.11575\left(\mathrm{C}_{22} \mathrm{H}_{21} \mathrm{O}_{10}\right.$, mass errors $4.23 \mathrm{ppm}$ and $4.35 \mathrm{ppm}$ ) in negative ion mode. DPIs, including [M-H-Glu] ${ }^{-}$at $\mathrm{m} / \mathrm{z} 283$ and $\left[\mathrm{M}-\mathrm{H}-\mathrm{Glu}-\mathrm{CH}_{3}\right]^{-}$at $\mathrm{m} / \mathrm{z} 268$, were also generated in their ESI-MS/MS spectra. By comparison with reference standards, $\mathbf{B} 9$ was positively determined to be calycosin-7-glucoside, while B20 was speculated to be a calycosin-7-glucoside isomer.

Two isomers, B16 and B22, which displayed [M-H] $]^{-}$ions at $\mathrm{m} / \mathrm{z} 431.09955$ and $\mathrm{m} / \mathrm{z} 431.09961$ $\left(\mathrm{C}_{21} \mathrm{H}_{19} \mathrm{O}_{10}\right.$, mass error $3.28 \mathrm{ppm}$ and $\left.3.42 \mathrm{ppm}\right)$, were eluted at 6.72 and $7.25 \mathrm{~min}$, respectively. They yielded ESI-MS ${ }^{2}$ product ions at $m / z 269$ [M-H-Glu] $^{-}$and $m / z 268$ [M-2H-Glu] $^{-}$. B16 was positively identified as genistin based on the comparison of the ESI-MS/MS spectra and retention time with reference standards. Meanwhile, $\mathbf{B} 22$ was predicted to be a genistin isomer.

B26 was eluted at $7.52 \mathrm{~min}$ with an $[\mathrm{M}-\mathrm{H}]^{-}$ion at $m / z 623.16339\left(\mathrm{C}_{28} \mathrm{H}_{31} \mathrm{O}_{16}\right.$, mass error $\left.4.379 \mathrm{ppm}\right)$. The $[\mathrm{M}-\mathrm{H}]^{-}$ion at $m / z 623$ generated characteristic fragment ions at $m / z 461, \mathrm{~m} / z 443$, and $m / z 299$. The former was generated from the neutral loss of glucose $(162 \mathrm{Da})$ from the $[\mathrm{M}-\mathrm{H}]^{-}$ion. The ion at $\mathrm{m} / z 461$ was further fragmented to yield fragment ions at $m / z 443$ and $m / z 299$ by neutral loss of $\mathrm{H}_{2} \mathrm{O}(18 \mathrm{Da}$ ) and glucose (162 Da). Hence, B26 was tentatively characterized as complanaruside.

In positive ion mode, $\mathbf{B} 37$ gave rise to $[\mathrm{M}+\mathrm{H}]^{+}$ions at $\mathrm{m} / \mathrm{z} 431.13263$ with a retention time of $8.26 \mathrm{~min}$. Its formula was speculated as $\mathrm{C}_{22} \mathrm{H}_{23} \mathrm{O}_{9}$ with a mass error of $-2.38 \mathrm{ppm}$. DPIs at $\mathrm{m} / z 269$ $[\mathrm{M}+\mathrm{H}-\mathrm{Glu}]^{+}$and $\mathrm{m} / z 413\left[\mathrm{M}+\mathrm{H}-\mathrm{Glu}-\mathrm{H}_{2} \mathrm{O}\right]^{+}$were observed. With the addition of standard substances, B37 was tentatively identified as ononin. 
Table 4. Chemical information of identified flavonoids in AR and FAR.

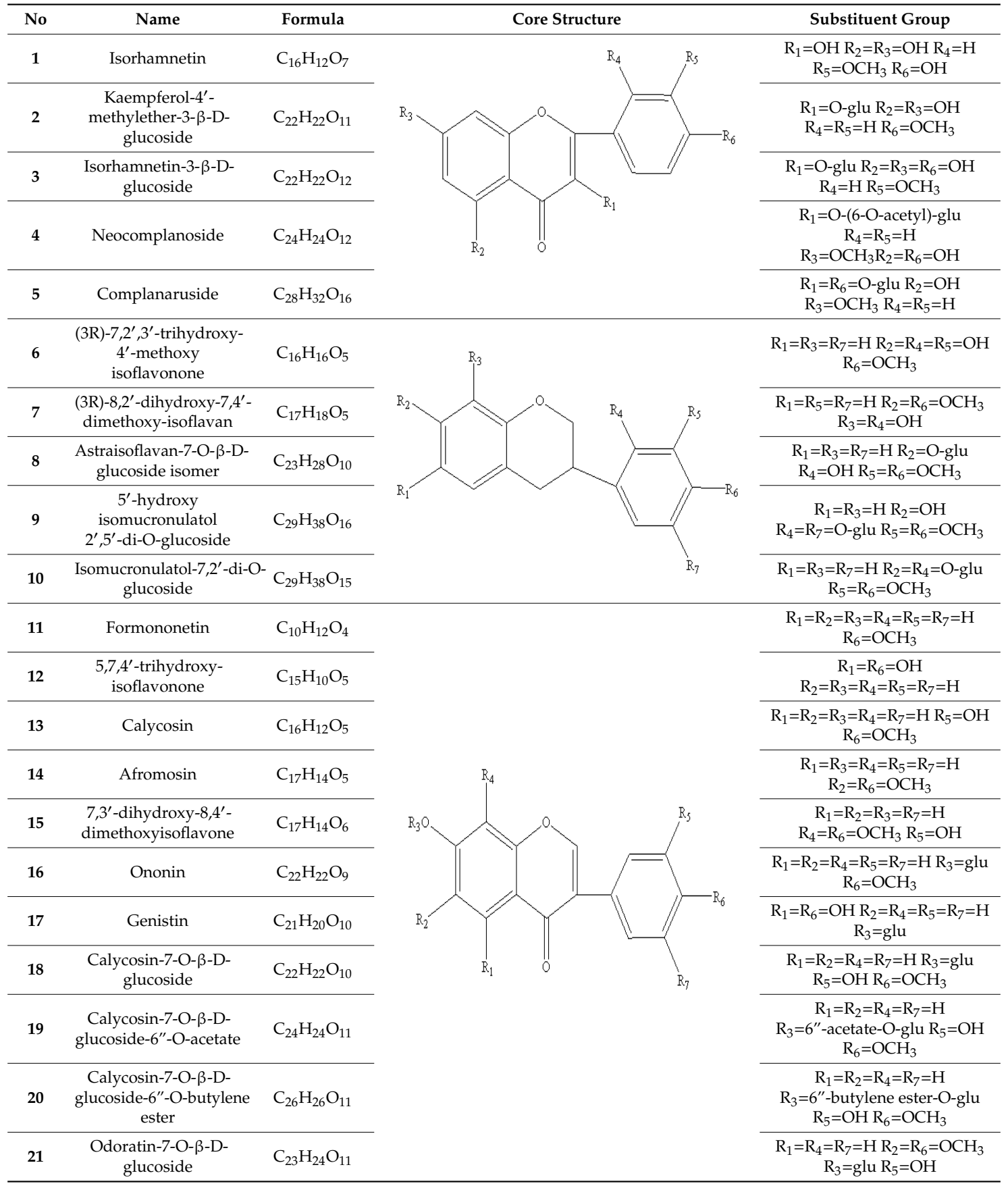

In negative ion mode, B26, which displayed [M-H] ${ }^{-}$ions at $m / z 429.11868\left(\mathrm{C}_{22} \mathrm{H}_{21} \mathrm{O}_{9}\right.$, mass error $1.565 \mathrm{ppm}$ ), was eluted at $13.38 \mathrm{~min}$. In the ESI-MS ${ }^{2}$ spectrum, it yielded product ions at $\mathrm{m} / \mathrm{z} 411$ $\left[\mathrm{M}-\mathrm{H}-\mathrm{H}_{2} \mathrm{O}\right]^{-}$and $m / z 267$ [M-H-Glc] $]^{-}$. According to the retention times of reference substances, B26 was unambiguously identified as ononin. Besides this, $\mathbf{B} 63$ was eluted at $12.20 \mathrm{~min}$ with $[\mathrm{M}-\mathrm{H}]^{-}$ ions at $m / z 315.05081\left(\mathrm{C}_{16} \mathrm{H}_{11} \mathrm{O}_{7}\right.$, mass error $\left.2.79 \mathrm{ppm}\right)$. On account of the neutral losses of $\mathrm{CH}_{2}$ and $\mathrm{CH}_{3}$, DPIs at $m / z 301$ and $m / z 300$ were respectively generated in its ESI-MS ${ }^{2}$ spectrum, which suggested the presence of a methoxy group. From the abovementioned analysis, B63 could be deduced as isorhamnetin.

B31, B36, B43, B46, B51, B61, and B64 were all observed with the same $[\mathrm{M}-\mathrm{H}]^{-}$ions at $m / z 267.06683$ $\left(\mathrm{C}_{16} \mathrm{H}_{11} \mathrm{O}_{4}\right)$ with mass errors within $5 \mathrm{ppm}$. They all produced DPIs at $m / z 252\left[\mathrm{M}-\mathrm{H}-\mathrm{CH}_{3}\right]^{-}$and $\mathrm{m} / \mathrm{z}$ $253\left[\mathrm{M}-\mathrm{H}-\mathrm{CH}_{2}\right]^{-}$in the ESI-MS ${ }^{2}$ spectra, corresponding to the characteristic fragmentation pathways 
of a methoxy group. According to the standard references, compound $\mathbf{B} 64$ was unambiguously characterized as formononetin, while the others were tentatively predicted to be formononetin isomers.

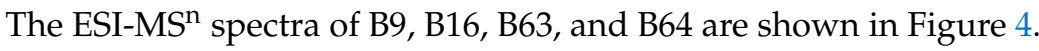
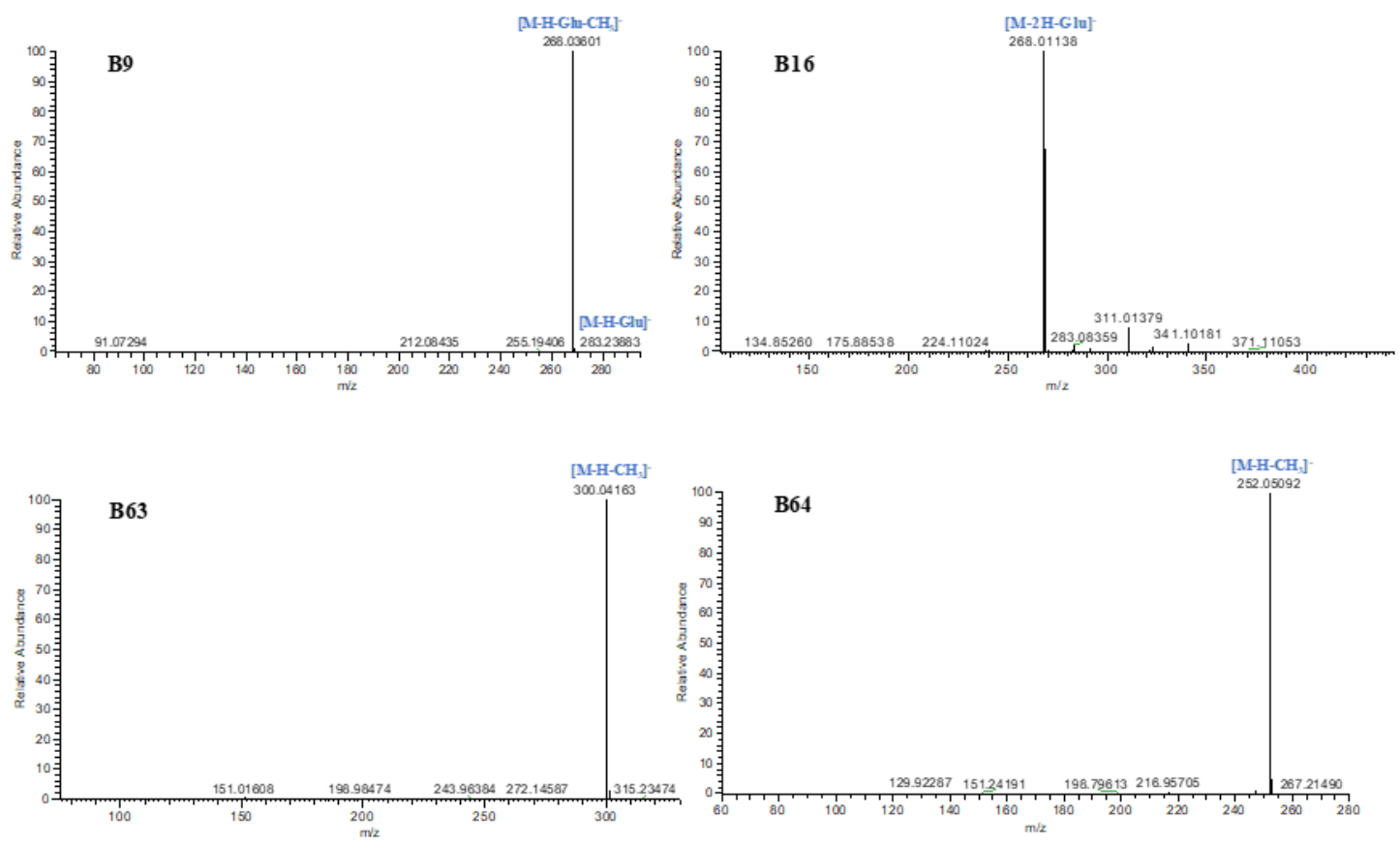

Figure 4. The ESI-MS ${ }^{\mathrm{n}}$ spectra of B9, B16, B63, and B64.

\subsubsection{Comparative Analysis of Constituents in AR and FAR}

To date, more than 100 compounds have been isolated and identified from AR. Saponins and flavonoids are considered the two most important constituents of AR for displaying bioactivities in vivo or in vitro [22]. Astragaloside I, isoastragaloside I, astragaloside II, isoastragaloside II, and astragaloside IV account for more than $80 \%$ of the total saponin content. It is noteworthy that variation of the saponin content among samples of different origins and parts, even in related preparations, is remarkable [23].

In our work, we found variation of saponins and flavonoids in FAR both in quality and amount, which was different from the former AR extract. The number of saponins species in FAR decreased from 42 to 29 , while quantities of certain saponins, such as isoastragaloside IV, increased with the process of fermentation. As above, the flavone aglycones reduced in number from 30 to 25 . The species and quantity of flavone glycosides changed obviously, even though the number was 17 in AR as well as in FAR (shown in Figure 5). 


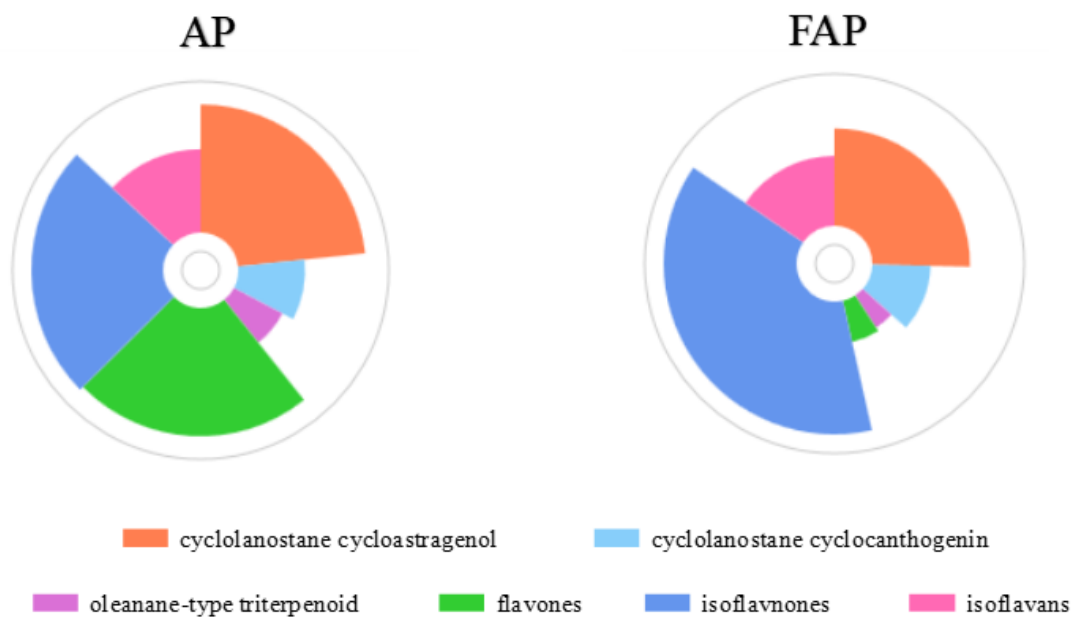

Figure 5. The classification of constituents in AR and FAR.

Moreover, the relative contents of some representative components changed greatly after fermentation (shown in Figure 6). In the process of fermentation, the contents of flavonoid glycosides-for instance, genistin, calycosin-7-glucoside, and complanaruside—dropped obviously. At the same time, the concentrations of certain saponins such as astragaloside I, astragaloside II, and isoastragaloside I decreased after fermentation, too. This result suggests that fermentation can accelerate the conversion of saponin glycosides into saponin aglycons and the hydrolysis of flavonoid glycosides to monoglycosides or aglycones. Besides this, owing to the presence of methoxyl groups, flavonoids were extremely unstable under the fermentation process. Thus, vast amounts of compounds may be altered into isomers during the fermentation process.
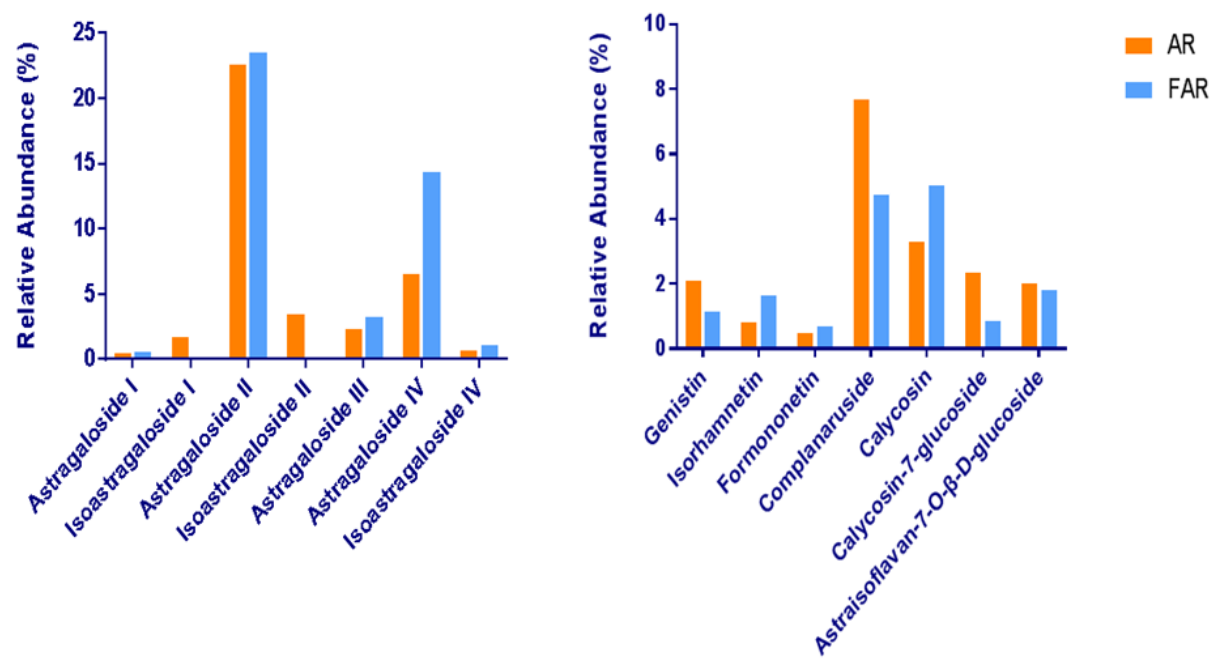

Figure 6. The changes in representative constituent contents in AR and FAR.

Noteworthily, the contents of astragaloside IV and isoastragaloside IV were significantly increased after fermentation, which means that the production of astragaloside IV was significantly higher than its consumption. It is also worth mentioning that astragaloside IV, noted for the quality control evaluation of AR in the Chinese Pharmacopeia, exhibits protective effects on cardiovascular disease, focal cerebral ischemia/reperfusion, liver cirrhosis, pulmonary disease, and diabetic nephropathy [24]. Although its content is relatively low in crude drugs, other astragalosides tend to be transformed into astragaloside IV in the fermentation process, which indicates that FAR may contribute to getting the necessary amount for the desired therapeutic effect. The probable transformations of astragaloside IV are illustrated in Figure 7. 


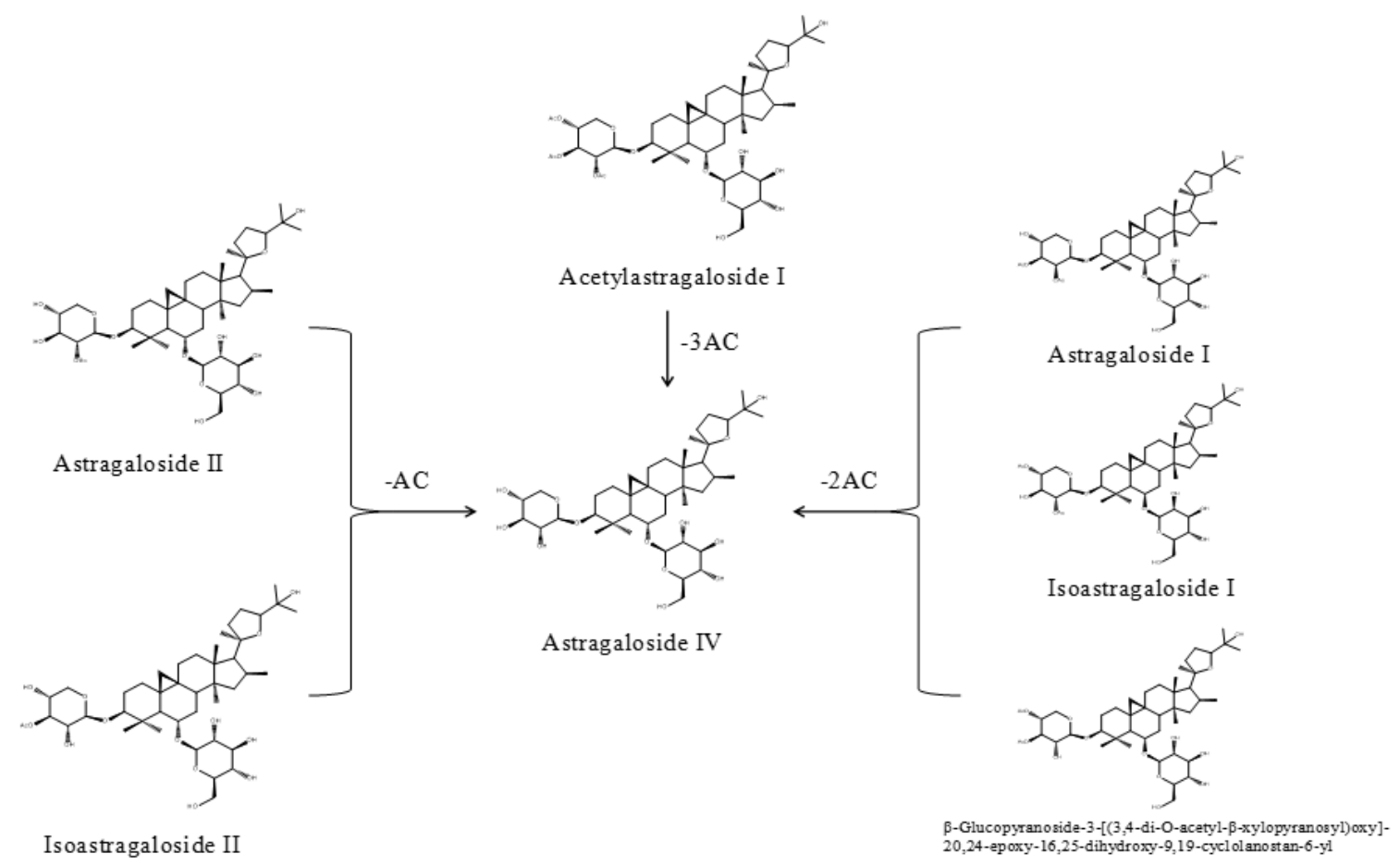

Figure 7. The probable transformations of astragaloside IV.

A few issues remain with this study. For example, fermentation induced a significant difference in compounds in FAR, but no specific transforming relationship was shown. The structures of newly generated constituents in AR by fermentation of Paecilomyces cicadae still remain obscure, but our findings encourage a much more in-depth analysis and structural elucidation.

\section{Conclusions}

In the present study, an effective strategy was established for the rapid screening and identification of target constituents in AR and FAR using FS-PIL-DE acquisition coupled to DPI analysis on a hybrid LTQ-Orbitrap MS in both positive and negative ion modes. A total of 107 compounds was preliminarily identified, including 42 saponins and 65 flavonoids. Our results indicated that AR fermentation with Paecilomyces significantly influenced the production of saponins and flavonoids. Among these compounds, the saponins were remarkedly reduced in connection with fermentation. This may be due to the degradation of saponins or flavonoid glycosides by hydrolytic enzymes, allowing the deglycosylated main backbone of glucoside to be divided into aglycone and oligosaccharides. This is the first study to show the changes in chemical components of unfermented AR and FAR, and it provides a foundation for further studies on the chemical interaction between microbiota and AR.

\section{Materials and Methods}

\subsection{Materials and Reagents}

Astragalus membranaceus (Fisch.) Bge. var. mongholicus (Bge.) Hsiao was obtained from Beijing Bencao Fangyuan Pharmaceutical Co., Ltd. (Beijing, China) and verified by Professor Yuan Zhang (Beijing University of Chinese Medicine, China). Paecilomyces cicadae (Miquel) Samson (No. cfcc81169) was provided by China Forestry Culture Collection Center (Beijing, China). Sixteen reference compounds, including astragaloside I, astragaloside II, astragaloside III, astragaloside IV, isoastragaloside I, isoastragaloside II, isoastragaloside IV, $\beta$-D-Glucopyranoside, $(3 \beta, 6 \alpha, 16 \beta, 20 \mathrm{R}$, 24S)-3-[(3,4-di-O-acetyl- $\beta$-D-xylopyranosyl)oxy]-20,24-epoxy-16,25-dihydroxy-9,19-cyclolanostan-6-yl, calycosin, calycosin-7-glucoside, formononetin, ononin, astraisoflavan-7-O $\beta$-D-glucoside, genistin, 
complanaruside, and isorhamnetin, were all purchased from Chengdu Must Biotechnology Co. Ltd. (Sichuan, China). Their structures were fully elucidated by comparing their spectra with the published literature. Their purities were acceptable ( $\geq 98 \%$ ) according to the requirements for HPLC-UV or HPLC-ELSD analysis.

HPLC-grade acetonitrile and formic acid (FA) were purchased from Thermo Fisher Scientific (Fair Lawn, NJ, USA). All other chemicals of analytical grade were available at the work station, Beijing Chemical Works (Beijing, China). Deionized water used throughout the experiment was purified by a Milli-Q Gradient Å 10 System (Millipore, Billerica, MA, USA). Grace Pure ${ }^{\mathrm{TM}}$ SPE C 18 -Low solid-phase extraction (SPE) cartridges (200 mg/3 mL, 59 m, $70 \AA$ ) were purchased from Grace Davison Discovery Science (Deerfield, IL, USA).

\subsection{Fermentation of $A R$}

AR was hot-air-dried for 2 days and then ground into a powder through a 100-mesh screen form using a blender. Laboratory-scale fermentation using AR was carried out in a $500 \mathrm{~mL}$ shake flask with a $250 \mathrm{~mL}$ working volume including $50 \mathrm{~g}$ of AR powder. A quantity of $50 \mathrm{~g}$ of AR powder was dissolved with $250 \mathrm{~mL}$ of distilled water and extracted at $121^{\circ} \mathrm{C}$ for $15 \mathrm{~min}$ by autoclaving. Paecilomyces cicadae (Miquel) Samson grown at 5\% $(v / v)$ in PDA liquid medium was used as an inoculum. The mixture was fermented at $28^{\circ} \mathrm{C}$ for 7 days on a rotatory shaker at $120 \mathrm{rpm} \cdot \mathrm{min}^{-1}$. Samples were taken on the 14 th day of fermentation for analyses. Unfermented AR was ground into a powder using a 100-mesh screen, inoculated into distilled water without Paecilomyces cicadae (Miquel) Samson, and cultured for 7 days at $28^{\circ} \mathrm{C}$ under aerobic conditions.

A volume of $1 \mathrm{~mL}$ of $\mathrm{AR}$ and FAR solution was added into an SPE cartridge pretreated with $5 \mathrm{~mL}$ methanol and $5 \mathrm{~mL}$ deionized water, in that order. Afterwards, the SPE cartridges were successively washed with $3 \mathrm{~mL}$ deionized water and $3 \mathrm{~mL}$ methanol, separately. The methanol eluate was evaporated to dryness by water bath at $70{ }^{\circ} \mathrm{C}$. Then, the residue was re-dissolved in $200 \mu \mathrm{L}$ methanol solution and centrifuged for $30 \mathrm{~min}\left(13,500 \mathrm{rpm}, 4^{\circ} \mathrm{C}\right)$. The supernatant was used for subsequent analysis.

\subsection{UHPLC-LTQ-Orbitrap MS Analysis}

\subsubsection{Instrument and Conditions}

UHPLC analysis was performed on a DIONEX Ultimate 3000 UHPLC system (Thermo Fisher Scientific, Waltham, MA, USA), equipped with a binary pump, an auto-sampler, a column compartment, and an electrospray ionization source. The chromatographic separation was carried out at $40{ }^{\circ} \mathrm{C}$ using a Waters ACQUITY HSS T3 column $(2.1 \times 100$ mm i.d., $1.8 \mu \mathrm{m}$; Waters Corporation, Milford, MA, USA). The mobile phase consisted of $0.1 \%$ FA aqueous solution (A) and acetonitrile (B) at a flow rate of $0.3 \mathrm{~mL} / \mathrm{min}$, and the linear gradient procedure was as follows: $0-6 \mathrm{~min}, 8 \%-30 \% \mathrm{~B} ; 6-14 \mathrm{~min}, 30 \%-40 \%$ B; $14-20.5 \mathrm{~min}, 40 \%-50 \% \mathrm{~B} ; 20.5-26 \mathrm{~min}, 30 \%-40 \% \mathrm{~B} ; 26-30 \mathrm{~min}, 40 \%-95 \% \mathrm{~B}$. The injection volume was $2 \mu \mathrm{L}$.

HRMS and MS/MS spectra were obtained using LTQ-Orbitrap MS with optimized operating parameters set as follows. Positive ion mode: sheath gas (nitrogen) flow rate of 40 arb, auxiliary gas (nitrogen) flow rate of $20 \mathrm{arb}$, capillary temperature of $350{ }^{\circ} \mathrm{C}$, spray voltage of $4.0 \mathrm{kV}$, capillary voltage of $25 \mathrm{~V}$, tube lens voltage of $110 \mathrm{~V}$. Negative ion mode: sheath gas (nitrogen) flow rate of 40 arb, auxiliary gas (nitrogen) flow rate of $20 \mathrm{arb}$, capillary temperature of $350{ }^{\circ} \mathrm{C}$, spray voltage of $3.0 \mathrm{kV}$, capillary voltage of $-35 \mathrm{~V}$, tube lens voltage of $-110 \mathrm{~V}$. The metabolites were detected by full-scan mass analysis from $\mathrm{m} / \mathrm{z} 100$ to $\mathrm{m} / \mathrm{z} 1200$ with a resolution of 30,000 in positive and negative ion modes. The collision energy for collision induced dissociation (CID) was adjusted to $40 \%$ of the maximum. Dynamic exclusion (DE) was used to prevent duplication. The repeat count was set to 5, and the dynamic repeat time was $30 \mathrm{~s}$ with a dynamic exclusion duration of $60 \mathrm{~s}$. In addition, $\mathrm{MS}^{\mathrm{n}}$ stages of the obtained datasets were employed using the PIL-DE dependent acquisition mode. 


\subsubsection{Data Processing}

A Thermo Xcalibur 2.1 (Thermo Scientific) workstation was used for data acquisition and data processing. In order to acquire as many fragment ions as possible, we selected the peaks with intensity over 10,000 for negative ion mode and over 40,000 for positive ion mode to identify components in AR and FAR. Based on the accurate mass, potential element compositions, and occurrence of possible reactions, the predicted atoms for chemical formulae of all the deprotonated and protonated molecular ions were set as follows: C [0-50], H [0-90], O [0-30], and ring double bond (RDB) equivalent value [0-15]. The maximum mass errors between the measured and calculated values were fixed within $5 \mathrm{ppm}$. All the relevant data, including peak number, retention time, accurate mass, the predicted chemical formula, and corresponding mass error, were recorded.

Supplementary Materials: Figure S1: Proposed fragmentation pathways for representative flavonoids detected in negative ion mode; Figure S2: Proposed fragmentation pathways for representative saponin detected in negative ion mode.

Author Contributions: J.Z. conceived and designed the experiments; L.D. supervised the experimental plan; X.M., W.S., Z.G. and Z.L. performed the experiments; Y.W., X.Z. and J.L. analyzed the data; Y.W. wrote the paper; J.Z. and L.D. reviewed the manuscript; all authors read and approved the final manuscript.

Funding: This work was financially supported by the Beijing Nova Program (Z171100001117029) and the Beijing University of Chinese Medicine Fund for Distinguished Young Scholars (No. 2018-JYB-XJ008).

Conflicts of Interest: The authors declare no conflict of interest.

\section{References}

1. $\mathrm{Xu}, \mathrm{L} . ; \mathrm{Du}, \mathrm{B} . ; \mathrm{Xu}$, B.J.F.C. A systematic, comparative study on the beneficial health components and antioxidant activities of commercially fermented soy products marketed in China. J. Food Chem. 2015, 174, 202-213. [CrossRef] [PubMed]

2. Stanton, C.; Ross, R.P.; Fitzgerald, G.F.; Sinderen, D.V. Fermented functional foods based on probiotics and their biogenic metabolites. J. Curr. Opin. Biotechnol. 2005, 16, 198-203. [CrossRef] [PubMed]

3. Yang, H.J.F.S. Study on Submerged Fermentation of Ganoderma lucidum into Chinese Pharmaceatical Edible Prodct. J. Food Chem. 2003, 24, 99-102.

4. Hussain, A.; Bose, S.; Wang, J.H.; Yadav, M.K.; Mahajan, G.B.; Kim, H. Fermentation, a feasible strategy for enhancing bioactivity of herbal medicines. J. Food Res. Int. 2016, 81, 1-16. [CrossRef]

5. Wang, Y.; He, P.; He, L.; Huang, Q.; Cheng, J.; Li, W.; Liu, Y.; Wei, C. Structural elucidation, antioxidant and immunomodulatory activities of a novel heteropolysaccharide from cultured Paecilomyces cicadae (Miquel.) Samson. J. Carbohydr. Polym. 2019, 216, 270-281. [CrossRef] [PubMed]

6. Ke, B.J.; Lee, C.L. Cordyceps cicadae NTTU 868 mycelium prevents $\mathrm{CCl}_{4}$-induced hepatic fibrosis in BALB/c mice via inhibiting the expression of pro-inflammatory and pro-fibrotic cytokines. J. Funct. Foods 2018, 43, 214-223. [CrossRef]

7. Wang, J.; Nie, S.; Cui, S.W.; Wang, Z.; Phillips, A.O.; Phillips, G.O.; Li, Y.; Xie, M. Structural characterization and immunostimulatory activity of a glucan from natural Cordyceps sinensis. J. Food Hydrocoll. 2017, 67, 139-147. [CrossRef]

8. Fu, J.; Wang, Z.; Huang, L.; Zheng, S.; Wang, D.; Chen, S.; Zhang, H.; Yang, S. Review of the botanical characteristics, phytochemistry, and pharmacology of Astragalus membranaceus (Huangqi). J. Phytother. Res. 2015, 28, 1275-1283. [CrossRef] [PubMed]

9. Cho, W.C.; Leung, K.N. In vitro and in vivo anti-tumor effects of Astragalus membranaceus. J. Cancer Lett. 2007, 252, 43-54. [CrossRef] [PubMed]

10. Liu, P.; Haiping, Z.; Yumin, L. Anti-Aging Implications of Astragalus Membranaceus (Huangqi): A Well-Known Chinese Tonic. J. Aging Dis. 2017, 8, 868. [CrossRef]

11. Lv, Y.W.; Hu, W.; Wang, Y.L.; Huang, L.F.; He, Y.B.; Xie, X.Z. Identification and determination of flavonoids in astragali radix by high performance liquid chromatography coupled with DAD and ESI-MS detection. J. Mol. 2011, 16, 2293. [CrossRef] [PubMed] 
12. Lyu, M.; Wang, Y.F.; Fan, G.W.; Wang, X.Y.; Xu, S.Y.; Zhu, Y. Balancing Herbal Medicine and Functional Food for Prevention and Treatment of Cardiometabolic Diseases through Modulating Gut Microbiota. J. Front. Microbiol. 2017, 8, 2146. [CrossRef] [PubMed]

13. Li, S.; Wu, D.; Lv, G.; Zhao, J. Carbohydrates analysis in herbal glycomics. J. Trends Anal. Chem. 2013, 52, 155-169. [CrossRef]

14. Kim, B.G.; Shin, K.S.; Yoon, T.J.; Yu, K.W.; Ra, K.S.; Kim, J.M.; Kim, S.Y.; Suh, H.J. Fermentation of Korean Red Ginseng byLactobacillus plantarumM-2 and Its Immunological Activities. J. Appl. Biochem. Biotechnol. 2011, 165, 1107-1119. [CrossRef] [PubMed]

15. Zhang, J.Y.; Wang, Z.J.; Zhang, Q.; Wang, F.; Ma, Q.; Lin, Z.Z.; Lu, J.Q.; Qiao, Y.J. Rapid screening and identification of target constituents using full scan-parent ions list-dynamic exclusion acquisition coupled to diagnostic product ions analysis on a hybrid LTQ-Orbitrap mass spectrometer. J. Talanta 2014, 124, 111-122. [CrossRef] [PubMed]

16. Zhang, J.Y.; Wang, Z.J.; Li, Y.; Liu, Y.; Cai, W.; Li, C.; Lu, J.Q.; Qiao, Y.J. A strategy for comprehensive identification of sequential constituents using ultra-High-performance liquid chromatography coupled with linear ion trap-Orbitrap mass spectrometer, application study on chlorogenic acids in Flos Lonicerae Japonicae. J. Talanta 2016, 147, 16-27. [CrossRef] [PubMed]

17. Shang, Z.; Cai, W.; Cao, Y.; Wang, F.; Wang, Z.; Lu, J.; Zhang, J. An integrated strategy for rapid discovery and identification of the sequential piperine metabolites in rats using ultra high-performance liquid chromatography/high resolution mass spectrometery. J. Pharm. Biomed. Anal. 2017, 146, 387. [CrossRef]

18. Liang, Y.; Zhao, W.; Wang, C.; Wang, Z.; Wang, Z.B.; Zhang, J.Y. A Comprehensive Screening and Identification of Genistin Metabolites in Rats Based on Multiple Metabolite Templates Combined with UHPLC-HRMS Analysis. J. Mol. 2018, 23, 1862. [CrossRef]

19. Zhang, J.; Wang, F.; Cai, W.; Zhang, Q.; Liu, Y.; Li, Y.; Liu, R.; Cao, G. Identification of metabolites of gardenin $\mathrm{A}$ in rats by combination of high-performance liquid chromatography with linear ion trap-Orbitrap mass spectrometer based on multiple data processing techniques. J. Biomed. Chromatogr. 2015, 29, 379-387. [CrossRef]

20. Wang, S. Fragment ion diagnostic strategies for the comprehensive identification of chemical profile of Gui-Zhi-Tang by integrating high-resolution MS, multiple-stage MS and UV information. J. Pharm. Biomed. Anal. 2014, 98, 22-35. [CrossRef]

21. Zhao, W.; Shang, Z.; Li, Q.; Huang, M.; He, W.; Wang, Z.; Zhang, J. Rapid Screening and Identification of Daidzein Metabolites in Rats Based on UHPLC-LTQ-Orbitrap Mass Spectrometry Coupled with Data-Mining Technologies. J. Mol. 2018, 23, 151. [CrossRef] [PubMed]

22. Chu, C.; Qi, L.W.; Li, B.; Gao, W.; Li, P. Radix Astragali (Astragalus): Latest Advancements and Trends in Chemistry, Analysis, Pharmacology and Pharmacokinetics. J. Curr. Org. Chem. 2010, 14. [CrossRef]

23. Yu, Q.T.; Qi, L.W.; Li, P.; Yi, L.; Zhao, J.; Bi, Z. Determination of seventeen main flavonoids and saponins in the medicinal plant Huang-qi (Radix astragali) by HPLC-DAD-ELSD. J. Sep. Sci. 2015, 30, 1292-1299. [CrossRef] [PubMed]

24. Liu, Z.H.; Liu, H.B.; Wang, J. Astragaloside IV protects against the pathological cardiac hypertrophy in mice. J. Biomed. Pharmacother. 2018, 97, 1468-1478. [CrossRef] [PubMed]

(C) 2019 by the authors. Licensee MDPI, Basel, Switzerland. This article is an open access article distributed under the terms and conditions of the Creative Commons Attribution (CC BY) license (http://creativecommons.org/licenses/by/4.0/). 